\title{
ON THE SUBANALYTICITY OF CARNOT-CARATHEODORY DISTANCES
}

\author{
Andrei AGRACHEV ${ }^{\mathrm{a}, \mathrm{b}}$, Jean-Paul GAUTHIER ${ }^{\mathrm{c}}$ \\ ${ }^{a}$ S.I.S.S.A., Via Beirut 2-4, 34013 Trieste, Italy

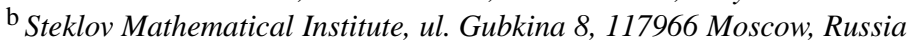 \\ ${ }^{\mathrm{c}}$ Lab. d'analyse appliquée et optimisation, Univ. de Bourgogne, Département de Mathématiques, \\ B.P. 47870, 21078 Dijon, France
}

Received 15 March 2000, revised 29 July 2000

\section{Introduction}

Let $M$ be a $C^{\infty}$ Riemannian manifold, $\operatorname{dim} M=n$. A distribution on $M$ is a smooth linear subbundle $\Delta$ of the tangent bundle $T M$. We denote by $\Delta_{q}$ the fiber of $\Delta$ at $q \in M ; \Delta_{q} \subset T_{q} M$. The number $k=\operatorname{dim} \Delta_{q}$ is the rank of the distribution. We assume that $1<k<n$. The restriction of the Riemannian structure to $\Delta$ is a sub-Riemannian structure.

Lipschitz integral curves of the distribution $\Delta$ are called admissible paths; these are Lipschitz curves $t \mapsto q(t), t \in[0,1]$, such that $\dot{q}(t) \in \Delta_{q(t)}$ for almost all $t$.

We fix a point $q_{0} \in M$ and study only admissible paths starting from this point, i.e. meeting the initial condition $q(0)=q_{0}$. Sections of the linear bundle $\Delta$ are smooth vector fields; we set

$$
\bar{\Delta}=\left\{X \in \operatorname{Vec} M: X(q) \in \Delta_{q}, q \in M\right\},
$$

the space of sections of $\Delta$. Iterated Lie brackets of the fields in $\bar{\Delta}$ define a flag

$$
\Delta_{q_{0}} \subset \Delta_{q_{0}}^{2} \subset \cdots \subset \Delta_{q_{0}}^{m} \cdots \subset T_{q} M
$$

in the following way:

$$
\Delta_{q_{0}}^{m}=\operatorname{span}\left\{\left[X_{1},\left[X_{2},\left[\ldots, X_{m}\right] \ldots\right]\left(q_{0}\right): X_{i} \in \bar{\Delta}, i=1, \ldots, m\right\} .\right.
$$

A distribution $\Delta$ is bracket generating at $q_{0}$ if $\Delta_{q_{0}}^{m}=T_{q_{0}} M$ for some $m>0$. If $\Delta$ is bracket generating, then according to the classical Rashevski-Chow theorem (see [11, 18]) there exist admissible paths connecting $q_{0}$ with any point of an open neighborhood of $q_{0}$. Moreover, applying a general existence theorem for optimal controls [12] one 
obtains that for any $q_{1}$ in a small enough neighborhood of $q_{0}$ there exists a shortest admissible path connecting $q_{0}$ to $q_{1}$. The Riemannian length of this shortest path is the sub-Riemannian distance or Carnot-Caratheodory distance between $q_{0}$ and $q_{1}$.

In the remainder of the paper we assume that $\Delta$ is bracket generating at the given initial point $q_{0}$. We denote by $\rho(q)$ the sub-Riemannian distance between $q_{0}$ and $q$. It follows from the Rashevsky-Chow theorem that $\rho$ is a continuous function defined on a neighborhood of $q_{0}$. Moreover, $\rho$ is Hölder-continuous with the Hölder exponent $1 / m$, where $\Delta_{q_{0}}^{m}=T_{q_{0}} M$.

We study mainly the case of real-analytic $M$ and $\Delta$. The germ at $q_{0}$ of a Riemannian distance is the square root of an analytic germ. This is not true for a sub-Riemannian distance function $\rho$. Moreover, $\rho$ is never smooth in a punctured neighborhood of $q_{0}$ (i.e. in a neighborhood without the pole $q_{0}$ ). It may happen that $\rho$ is not even subanalytic. The main results of the paper concern subanalyticity properties of $\rho$ in the case of a generic real-analytic $\Delta$.

We prove that, generically, the germ of $\rho$ at $q_{0}$ is subanalytic if:

$$
n \leqslant(k-1) k+1 \quad \text { (Theorem } 7),
$$

and is not subanalytic if:

$$
n \geqslant(k-1)\left(\frac{k^{2}}{3}+\frac{5 k}{6}+1\right) \quad(\text { Theorem 10). }
$$

The balls $\rho^{-1}([0, r])$ of small enough radius are subanalytic if $n>k \geqslant 3$ (Theorem 9). This statement about the balls is valid not only generically, but up to a set of distributions of codimension $\infty$.

In particular, if $k \geqslant 3, n \geqslant(k-1)\left(\frac{k^{2}}{3}+\frac{5 k}{6}+1\right)$, then (generically!) the balls $\rho^{-1}([0, r])$ are subanalytic but $\rho$ is not!

This paper is a new step in a rather long research line, see $[1,5,6,9,10,15,17,20]$. The main tools are the nilpotent approximation, Morse-type indices of geodesics, both in the normal and abnormal cases, and transversality techniques.

We finish the introduction with some conjectures on still open questions.

(1) Small balls $\rho^{-1}([0, r])$ for $k=2, n \geqslant 4$. A natural conjecture is that they are, generically, not subanalytic.

(2) The germ of $\rho$ at $q_{0}$ for $(k-1) k+1<n<(k-1)\left(\frac{k^{2}}{3}+\frac{5 k}{6}+1\right)$. The bound $n \leqslant(k-1) k+1$ for "generically subanalytic dimensions" is, perhaps, exact, while the bound $n \geqslant(k-1)\left(\frac{k^{2}}{3}+\frac{5 k}{6}+1\right)$ for "generically nonsubanalytic dimensions" may, probably, be improved. For a wide range of dimensions, the subanalyticity and nonsubanalyticity of the germ of $\rho$ should be both typical (i.e. valid for open sets of real-analytic distributions).

\section{Nilpotentization}

Nilpotentization or nilpotent approximation is a fundamental operation in the geometric control theory and sub-Riemannian geometry; this is a real nonholonomic analog of the usual linearization (see $[2,3,7,8,19])$. 
Given nonnegative integers $k_{1}, \ldots, k_{l}$, where $k_{1}+\cdots+k_{l}=n$, we present $\mathbb{R}^{n}$ as a direct sum $\mathbb{R}^{k_{1}} \oplus \cdots \oplus \mathbb{R}^{k_{l}}$. Any vector $x \in \mathbb{R}^{n}$ takes the form

$$
x=\left(x_{1}, \ldots, x_{l}\right), \quad x_{i}=\left(x_{i 1}, \ldots, x_{i k_{i}}\right) \in \mathbb{R}^{k_{i}}, \quad i=1, \ldots, l .
$$

The differential operators on $\mathbb{R}^{n}$ with smooth coefficients have the form

$$
\sum_{\alpha} \frac{a_{\alpha}(x) \partial^{|\alpha|}}{\partial x^{\alpha}}
$$

where $a_{\alpha} \in C^{\infty}\left(\mathbb{R}^{n}\right)$ and $\alpha$ is a multi-index:

$$
\alpha=\left(\alpha_{1}, \ldots, \alpha_{l}\right), \quad \alpha_{i}=\left(\alpha_{i 1}, \ldots, \alpha_{i k_{i}}\right), \quad\left|\alpha_{i}\right|=\sum_{j=1}^{k_{i}} \alpha_{i j}, \quad i=1, \ldots, l .
$$

The space of all differential operators with smooth coefficients forms an associative algebra with composition of operators as multiplication. The differential operators with polynomial coefficients form a subalgebra of this algebra with generators $1, x_{i j}, \frac{\partial}{\partial x_{i j}}, i=$ $1, \ldots, l, j=1, \ldots, k_{i}$. We introduce a $\mathbb{Z}$-grading into this subalgebra by giving the weights $v$ to the generators: $v(1)=0, v\left(x_{i j}\right)=i$, and $v\left(\frac{\partial}{\partial x_{i j}}\right)=-i$. Accordingly,

$$
v\left(x^{\alpha} \frac{\partial^{|\beta|}}{\partial x^{\beta}}\right)=\sum_{i=1}^{l}\left(\left|\alpha_{i}\right|-\left|\beta_{i}\right|\right) i,
$$

where $\alpha$ and $\beta$ are multi-indices.

A differential operator with polynomial coefficients is said to be v-homogeneous of weight $m$ if all the monomials occurring in it have weight $m$. It is easy to see that $v\left(D_{1} \circ D_{2}\right)=v\left(D_{1}\right)+v\left(D_{2}\right)$ for any $v$-homogeneous differential operators $D_{1}$ and $D_{2}$. The most important for us are differential operators of order 0 (functions) and of order 1 (vector fields). We have $v(X a)=v(X)+v(a), v\left(\left[X_{1}, X_{2}\right]\right)=v\left(X_{1}\right)+v\left(X_{2}\right)$ for any $v$-homogeneous function $a$ and vector fields $X, X_{1}, X_{2}$. A differential operator of order $N$ has weight at least $-N l$; in particular, the weight of nonzero vector fields is at least $-l$. Vector fields of nonnegative weights vanish at 0 while the values at 0 of the fields of weight $-i$ belong to the subspace $\mathbb{R}^{k_{i}}$, the $i$ th summand in the presentation $\mathbb{R}^{n}=\mathbb{R}^{k_{1}} \oplus \cdots \oplus \mathbb{R}^{k_{l}}$.

We introduce a dilation $\delta_{t}: \mathbb{R}^{n} \rightarrow \mathbb{R}^{n}, t \in \mathbb{R}$, by the formula:

$$
\delta_{t}\left(x_{1}, x_{2}, \ldots, x_{l}\right)=\left(t x_{1}, t^{2} x_{2}, \ldots, t^{l} x_{l}\right) .
$$

$v$-homogeneity means homogeneity with respect to this dilation. In particular, we have $a\left(\delta_{t} x\right)=t^{\nu(a)} a(x), \delta_{t *} X=t^{-v(X)} X$ for any $v$-homogeneous function $a$ and vector field $X$.

Now let $X=\sum_{i, j} a_{i j} \frac{\partial}{\partial x_{i j}}$ be an arbitrary smooth vector field. Expanding the coefficients $a_{i j}$ in a Taylor series in powers of $x_{i j}$ and grouping the terms with the same weights, we get an expansion $X \approx \sum_{m=-l}^{+\infty} X^{(m)}$, where $X^{(m)}$ is a $v$-homogeneous field 
of weight $m$. This expansion enables us to introduce a decreasing filtration in the Lie algebra of smooth vector fields $\mathrm{Vec} \mathbb{R}^{n}$ by putting:

$$
\operatorname{Vec}^{m}\left(k_{1}, \ldots, k_{l}\right)=\left\{X \in \operatorname{Vec} \mathbb{R}^{n}: X^{(i)}=0 \text { for } i<m\right\}, \quad-l \leqslant m<+\infty .
$$

It is easy to see that:

$$
\left[\operatorname{Vec}^{m_{1}}\left(k_{1}, \ldots, k_{l}\right), \operatorname{Vec}^{m_{2}}\left(k_{1}, \ldots, k_{l}\right)\right] \subseteq \operatorname{Vec}^{m_{1}+m_{2}}\left(k_{1}, \ldots, k_{l}\right) .
$$

It happens that this class of filtrations is in a sense universal. We will need the following theorem which is a special case of general results proved in $[2,8]$.

Set $\Delta_{q_{0}}^{0}=\{0\}_{q_{0}}, \Delta_{q_{0}}^{1}=\Delta_{q_{0}}$.

THEOREM 1. - Assume that $\operatorname{dim}\left(\Delta_{q_{0}}^{i} / \Delta_{q_{0}}^{i-1}\right)=k_{i}, i=1, \ldots, l$. Then there exists $a$ neighborhood $O_{q_{0}}$ of the point $q_{0}$ in $M$ and a coordinate mapping $\chi: O_{q_{0}} \rightarrow \mathbb{R}^{n}$ such that

$$
\chi\left(q_{0}\right)=0,\left.\quad \chi_{*}\right|_{T_{q_{0}} M}\left(\Delta_{q_{0}}^{i}\right)=\mathbb{R}^{k_{1}} \oplus \cdots \oplus \mathbb{R}^{k_{i}}, \quad 1 \leqslant i \leqslant l,
$$

and $\chi_{*}(\bar{\Delta}) \subset \operatorname{Vec}^{-1}\left(k_{1}, \ldots, k_{l}\right)$.

The mapping $\chi: O_{q_{0}} \rightarrow \mathbb{R}^{n}$ from the theorem is called an adapted coordinate map. It is obtained from arbitrary coordinates by a polynomial change of variables and the construction is quite effective. For any $X \in \bar{\Delta}$ we have $\chi_{*}(X) \approx \chi_{*}(X)^{(-1)}+$ $\sum_{j \geqslant 0} \chi_{*}(X)^{(j)}$, where $\chi_{*}(X)^{(m)}$ is a $\nu$-homogeneous field of weight $m$. The field $\widehat{X}=$ $\chi_{*}^{-1}\left(\chi_{*}(X)^{(-1)}\right)$ is called the nilpotentization of $X$ (relative to the adapted coordinate mapping $\chi)$.

Proposition 1. - Assume that $\chi=\left(\chi_{1}, \ldots, \chi_{l}\right), \chi_{j}: O_{q_{0}} \rightarrow \mathbb{R}^{k_{j}}, j=1, \ldots, l$, is an adapted coordinate map, $X_{1}, \ldots, X_{i} \in \bar{\Delta}$, and $\widehat{X}_{l}$ is the nilpotenization of $X_{l}$, $\imath=1, \ldots, i$. Then:

$$
\begin{gathered}
X_{1} \circ \cdots \circ X_{i} \chi_{j}\left(q_{0}\right)=0 \quad \forall j>i, \\
X_{1} \circ \cdots \circ X_{i} \chi_{i}\left(q_{0}\right)=\widehat{X}_{1} \circ \cdots \circ \widehat{X}_{i} \chi_{i}\left(q_{0}\right) .
\end{gathered}
$$

Proof. - We have:

$$
\begin{aligned}
& X_{1} \circ \cdots \circ X_{i} \chi_{j}\left(q_{0}\right)=\left.\left(\chi_{*} X_{1}\right) \circ \cdots \circ\left(\chi_{*} X_{i}\right) x_{j}\right|_{0} \\
& \quad=\left.\sum_{m_{1}+\cdots+m_{i}=-j}\left(\chi_{*} X_{1}\right)^{\left(m_{1}\right)} \circ \cdots \circ\left(\chi_{*} X_{i}\right)^{\left(m_{i}\right)} x_{j}\right|_{0},
\end{aligned}
$$

since any monomial of positive weight vanishes at 0 . Hence:

$$
\begin{gathered}
X_{1} \circ \cdots \circ X_{i} \chi_{j}\left(q_{0}\right)=0 \text { for } i<j, \\
X_{1} \circ \cdots \circ X_{i} \chi_{i}\left(q_{0}\right)=\left.\left(\chi_{*} X_{1}\right)^{(-1)} \circ \cdots \circ\left(\chi_{*} X_{i}\right)^{(-1)} x_{i}\right|_{0}=\widehat{X}_{1} \circ \cdots \circ \widehat{X}_{i} \chi_{i}\left(q_{0}\right) .
\end{gathered}
$$




\section{The endpoint mapping}

We are working in a small neighborhood $O_{q_{0}}$ of $q_{0} \in M$, where we fix an orthonormal frame $X_{1}, \ldots, X_{k} \in \operatorname{Vec} O_{q_{0}}$ of the sub-Riemannian structure under consideration. Admissible paths are thus solutions of the Cauchy problem:

$$
\dot{q}=\sum_{i=1}^{k} u_{i}(t) X_{i}(q), \quad q \in O_{q_{0}}, q(0)=q_{0},
$$

where $u=\left(u_{1}(\cdot), \ldots, u_{k}(\cdot)\right) \in L_{2}^{k}[0,1]$.

Below $\|u\|=\left(\int_{0}^{1} \sum_{i=0}^{k} u_{i}^{2}(t) d t\right)^{1 / 2}$ is the norm in $L_{2}^{k}[0,1]$. We also set $\|q(\cdot)\|=\|u\|$, where $q(\cdot)=q(\cdot ; u)$ is the solution of (2). Let:

$$
U_{r}=\left\{u \in L_{2}^{k}[0,1]:\|u\|=r\right\},
$$

be the sphere of radius $r$ in $L_{2}^{k}[0,1]$. Solutions of (2) are defined for all $t \in[0,1]$, if $u$ belongs to a sphere of radius $r$, small enough. In this paper we implicitely take $u$ only in such spheres. The length $l(q(\cdot))=\int_{0}^{1}\left(\sum_{i=1}^{k} u_{i}^{2}(t)\right)^{1 / 2} d t$ is well-defined and satisfies the inequality:

$$
l(q(\cdot)) \leqslant\|q(\cdot)\|=r .
$$

The length does not depend on the parametrization of the curve while the norm $\|u\|$ depends. We say that $u$ and $q(\cdot)$ are normalized if $\sum_{i=1}^{k} u_{i}^{2}(t)$ does not depend on $t$. For normalized $u$, and only for them, the inequality (3) becomes an equality.

We consider the endpoint mapping $f: u \mapsto q(1)$. It is a well-defined smooth mapping of a neighborhood of the origin of $L_{2}^{k}[0,1]$ into $M$. Clearly, $\rho(q)=\min \{\|u\|: u \in$ $\left.L_{2}^{r}[0,1], f(u)=q\right\}$ and the minimum is attained at a normalized control. A normalized $u$ is called minimal for the system (2) if $\rho(f(u))=\|u\|$.

Remark. - The notations $\|q(\cdot)\|$ and $l(q(\cdot))$ reflect the fact that these quantities do not depend on the choice of the orthonormal frame $X_{1}, \ldots, X_{k}$ and are characteristics of the trajectory $q(\cdot)$ rather than the control $u$. The $L_{2}$-topology in the space of controls is the $H_{1}$-topology in the space of trajectories.

Let $\chi: O_{q_{0}} \rightarrow \mathbb{R}^{n}$, be an adapted coordinate map and $\widehat{X}_{i}$ be the nilpotentization of $X_{i}$, $i=1, \ldots, k$. The system:

$$
\dot{x}=\sum_{i=1}^{k} u_{i}(t) \chi_{*} \widehat{X}_{i}(x), \quad x \in \mathbb{R}^{n}, x(0)=0,
$$

is the nilpotentization of the system (2) expressed in the adapted coordinates.

We define the mapping $\hat{f}: L_{2}^{k}[0,1] \rightarrow \mathbb{R}^{n}$ by the rule $\hat{f}: u(\cdot) \mapsto x(1)$, where $x(\cdot)=$ $x(\cdot ; u)$ is the solution of $(\hat{2})$. The following proposition is an easy corollary of the fact that $\chi_{*} \widehat{X}_{i}$ are $\nu$-homogeneous of weight $(-1)$ (see [2] for details). 
Proposition 2. - Let $\chi=\left(\chi_{1}, \ldots, \chi_{l}\right), \chi_{j}: O_{q_{0}} \rightarrow \mathbb{R}^{k_{j}}, j=1, \ldots, l$. Then the following identities hold for any $u(\cdot) \in L_{2}^{k}[0,1], \varepsilon \in \mathbb{R}$ :

$$
\begin{aligned}
\hat{f}(u(\cdot))= & \left(\int_{0}^{1} \sum_{i=1}^{k} u_{i}(t) \widehat{X}_{i} \chi_{1}\left(q_{0}\right) d t, \ldots,\right. \\
& \left.\int_{0 \leqslant t_{1} \leqslant \cdots \leqslant t_{l} \leqslant 1} \ldots \sum_{i_{J}=1}^{k} u_{i_{1}}\left(t_{1}\right) \cdots u_{i_{l}}\left(t_{l}\right) \widehat{X}_{i_{1}} \circ \cdots \circ \widehat{X}_{i_{l}} \chi_{l}\left(q_{0}\right) d t_{1} \cdots d t_{l}\right) ;
\end{aligned}
$$

$\hat{f}(\varepsilon u(\cdot))=\delta_{\varepsilon} \hat{f}(u(\cdot))$, where $\delta_{\varepsilon}$ is the dilation (1).

We set $f_{\varepsilon}(u)=\delta_{\frac{1}{\varepsilon}} \chi(f(\varepsilon u))$. Then $f_{\varepsilon}$ is a smooth mapping from a neighborhood of 0 in $L_{2}^{k}[0,1]$ to $\mathbb{R}^{n}$. Moreover, any bounded subset of $L_{2}^{k}[0,1]$ is contained in the domain of $f_{\varepsilon}$ for $\varepsilon$ small enough.

THEOREM 2. $-f_{\varepsilon} \rightarrow \hat{f}$ as $\varepsilon \rightarrow 0$ in the $C^{\infty}$ topology of the uniform convergence of the mappings and all their derivatives on the balls in $L_{2}^{k}[0,1]$.

Proof. - We have:

$$
\begin{aligned}
& \delta_{\frac{1}{\varepsilon}} \chi(f(v))=\left(\frac{1}{\varepsilon} \chi_{1}(f(v)), \ldots, \frac{1}{\varepsilon^{l}} \chi_{l}(f(v))\right) \\
& \chi_{j}(f(v))=\int_{0}^{1} \sum_{i=1}^{k} v_{i}(t) X_{j} \chi_{j}(q(t)) d t=\int_{0}^{1} \sum_{i=1}^{k} v_{i}(t) X_{j} \chi_{j}\left(q_{0}\right) d t \\
&+\int_{0}^{1} \int_{0}^{t_{2}} \sum_{i_{1}=i_{2}=1}^{k} v_{i_{1}}\left(t_{1}\right) v_{i_{2}}\left(t_{2}\right) X_{i_{1}} \circ X_{i_{2}} \chi_{j}\left(q\left(t_{1}\right)\right) d t_{1} d t_{2} \\
&=\int_{0}^{1} \sum_{i=1}^{k} v_{i}(t) X_{j} \chi_{j}\left(q_{0}\right) d t \\
&+\int_{0}^{1} \int_{0}^{t_{2}} \sum_{i_{1}=i_{2}=1}^{k} v_{i_{1}}\left(t_{1}\right) v_{i_{2}}\left(t_{2}\right) X_{i_{1}} \circ X_{i_{2}} \chi_{j}\left(q_{0}\right) d t_{1} d t_{2} \\
&+\int_{0 \leqslant t_{1} \leqslant t_{2} \leqslant t_{3}} \int_{i_{j}=1}^{k} v_{i_{1}}\left(t_{1}\right) v_{i_{2}}\left(t_{2}\right) v_{i_{3}}\left(t_{3}\right) X_{i_{1}} \circ X_{i_{2}} \circ X_{i_{3}} \chi_{j}\left(q\left(t_{1}\right)\right) d t_{1} d t_{2} d t_{3} \\
&= \cdots
\end{aligned}
$$

Now, Proposition 1 implies:

$$
\frac{1}{\varepsilon^{j}} \chi_{j}(f(\varepsilon u))=\int_{0 \leqslant t_{1} \leqslant \cdots \leqslant t_{j} \leqslant 1} \cdots \sum_{i_{j}=1}^{k} u_{i_{1}}\left(t_{1}\right) \cdots u_{i_{j}}\left(t_{j}\right) \widehat{X}_{i_{1}} \circ \cdots \circ \widehat{X}_{i_{j}} \chi_{j}\left(q_{0}\right) d t_{1} \cdots d t_{j}
$$




$$
\begin{aligned}
& +\varepsilon \int_{0 \leqslant t_{1} \leqslant \cdots \leqslant t_{j+1} \leqslant 1} \cdots \sum_{i_{j}=1}^{k} u_{i_{1}}\left(t_{1}\right) \cdots u_{i_{j+1}}\left(t_{j+1}\right) X_{i_{1}} \circ \cdots \\
& \circ X_{i_{j+1}} \chi_{j}\left(q\left(t_{1} ; \varepsilon u\right)\right) d t_{1} \cdots d t_{j+1} .
\end{aligned}
$$

It remains to apply Proposition 2 and to note that the mappings $v \mapsto q(t ; v)$ are uniformly bounded with all their derivatives on a small enough ball in $L_{2}^{k}[0,1]$ for $0 \leqslant t \leqslant 1$.

Recall that $\rho(q)=\min \left\{\|u\|: f(u)=q, u \in L_{2}^{k}[0,1]\right\}$ is the sub-Riemannian distance function. We set:

$$
\rho_{\varepsilon}(x)=\min \left\{\|u\|: f_{\varepsilon}(u)=x, u \in L_{2}^{k}[0,1]\right\}=\frac{1}{\varepsilon} \rho\left(\chi^{-1}\left(\delta_{\varepsilon} x\right)\right)
$$

and

$$
\hat{\rho}(x)=\min \left\{\|u\|: \hat{f}(u)=x, u \in L_{2}^{k}[0,1]\right\} .
$$

Thus $\hat{\rho}$ is the sub-Riemannian distance for the nilpotentization of the original system.

LEMMA 1. - The family of functions $\left.\rho_{\varepsilon}\right|_{K}$ is equicontinuous for any compact $K \subset$ $\mathbb{R}^{n}$.

Proof. - The function $\rho(q)$ is the sub-Riemannian distance between $q_{0}$ and $q$ for the sub-Riemannian structure with the orthonormal frame $X_{1}, \ldots, X_{k}$. Hence $\rho_{\varepsilon}(x)$ is the sub-Riemannian distance between 0 and $x$ for the structure with the orthonormal frame:

$$
\varepsilon\left(\delta_{\varepsilon}^{-1}\right)_{*} \chi_{*} X_{1}, \ldots, \varepsilon\left(\delta_{\varepsilon}^{-1}\right)_{*} \chi_{*} X_{k} .
$$

Let $d_{\varepsilon}(x, y)$ be the distance between $x$ and $y$ for this sub-Riemannian structure so that $\rho_{\varepsilon}(x)=d_{\varepsilon}(0, x)$. Clearly, $\left|\rho_{\varepsilon}(x)-\rho_{\varepsilon}(y)\right| \leqslant d_{\varepsilon}(x, y)$. We are going to prove that:

$$
d_{\varepsilon}(x, y) \leqslant c|x-y|^{1 / 2^{l}} .
$$

First we introduce an auxiliary operation on families of control functions. Suppose that $u_{s}(\cdot), v_{s}(\cdot) \in L_{2}^{k}[0,1], s \in \mathbb{R}, u_{0}(\cdot)=v_{0}(\cdot)=0$; we define:

$$
[u, v]_{s}(t)= \begin{cases}u_{|s|^{1 / 2}}(4 t), & 0 \leqslant t<\frac{1}{4}, \\ v_{|s|^{1 / 2}}(4 t-1), & \frac{1}{4} \leqslant t<\frac{1}{2}, \\ u_{|s|^{1 / 2}}(3-4 t), & \frac{1}{2} \leqslant t<\frac{3}{4}, \\ v_{|s|^{1 / 2}}(4-4 t), & \frac{3}{4} \leqslant t \leqslant 1,\end{cases}
$$

where we take a branch of $|s|^{1 / 2}$ such that $s|s|^{1 / 2} \geqslant 0$.

For any control $u(\cdot)$ and a system:

$$
\dot{x}=\sum_{i=1}^{k} u_{i}(t) Z_{i}(x), \quad x \in \mathbb{R}^{n},
$$


we define a diffeomorphism $\mathfrak{Z}_{u}: \mathbb{R}^{n} \rightarrow \mathbb{R}^{n}$ by the rule $\mathfrak{Z}_{u}(x(0))=x(1)$, where $t \mapsto x(t)$ is a solution of the differential equation (5). Then

$$
\mathfrak{Z}_{[u, v]_{s}}=\mathfrak{Z}_{v_{|s|^{1 / 2}}}^{-1} \circ \mathfrak{Z}_{u_{|s|}-1 / 2}^{-1} \circ \mathfrak{Z}_{v_{|s| 1 / 2}} \circ \mathfrak{Z}_{\left.u_{|s|}\right|^{1 / 2}}
$$

If $(s, x) \mapsto \mathfrak{Z}_{u_{s}}(x),(s, x) \mapsto \mathfrak{Z}_{v_{s}}(x)$ are $C^{1}$-mappings and $\left.\frac{\partial}{\partial s} \mathfrak{Z}_{u_{s}}\right|_{s=0}=X,\left.\frac{\partial}{\partial s} \mathfrak{Z}_{v_{s}}\right|_{s=0}=Y$, $X, Y \in \operatorname{Vec} \mathbb{R}^{n}$, then $(s, x) \mapsto \mathfrak{Z}_{[u, v]_{s}}(x)$ is also $C^{1}$ and $\left.\frac{\partial}{\partial s} \mathfrak{Z}_{[u, v]_{s}}\right|_{s=0}=[X, Y]$. Let $\varsigma_{s}^{i}$ be the constant control with the $i$ th coordinate equals $s$ and all other coordinates equals 0 . We set $\varsigma\left[i_{1} \ldots i_{m}\right]_{s}=\left[\varsigma^{i_{1}},\left[\ldots, \varsigma^{i_{m}}\right] \ldots\right]_{s}$ and obtain $\left.\frac{\partial}{\partial s} \mathfrak{Z}_{\varsigma\left[i_{1} \ldots i_{m}\right]_{s}}\right|_{s=0}=$ $\left[Z_{1},\left[\ldots, Z_{m}\right] \ldots\right]$. Note that $\left\|s\left[i_{1} \ldots i_{m}\right]_{s}\right\|=s^{1 / 2^{m}}$.

Now we go back to the vector fields (4) and set $Z_{i}^{\varepsilon}=\varepsilon \delta_{\varepsilon *}^{-1} \chi_{*} X_{i}, i=1, \ldots, k$. We have $\delta_{\varepsilon *}^{-1} \chi_{*} X_{i}=\frac{1}{\varepsilon} \chi_{*} \widehat{X}_{i}+R_{i}^{\varepsilon}$, where $R_{i}^{\varepsilon}$ is a family of vector fields smooth with respect to $\varepsilon$ (see Section 2). Hence $Z_{i}^{\varepsilon}=\chi_{*} \widehat{X}_{i}+\varepsilon R_{i}^{\varepsilon}$.

The bracket generating assumption implies that a basis of $\mathbb{R}^{n}$ can be formed by vectors:

$$
\left[X_{i_{1}^{1}},\left[\ldots, X_{i_{m_{1}}^{1}}\right] \ldots\right]\left(q_{0}\right), \ldots,\left[X_{i_{1}^{n}},\left[\ldots, X_{i_{m_{n}}^{n}}\right] \ldots\right]\left(q_{0}\right),
$$

where $1 \leqslant m_{1} \leqslant \cdots \leqslant m_{n} \leqslant l$. It follows from Proposition 1 that the vectors:

$$
\left[\widehat{X}_{i_{1}^{1}},\left[\ldots, \widehat{X}_{i_{m_{1}}^{1}}\right] \ldots\right]\left(q_{0}\right), \ldots,\left[\widehat{X}_{i_{1}^{n}},\left[\ldots, \widehat{X}_{i_{m_{n}}^{n}}\right] \ldots\right]\left(q_{0}\right),
$$

form a basis of $\mathbb{R}^{n}$. Indeed, the difference:

$$
\left[X_{i_{1}^{j}},\left[\ldots, X_{i_{m_{j}}^{j}}\right] \ldots\right]\left(q_{0}\right)-\left[\widehat{X}_{i_{1}^{j}},\left[\ldots, \widehat{X}_{i_{m_{j}}^{j}}\right] \ldots\right]\left(q_{0}\right),
$$

belongs to $\Delta_{q_{0}}^{m_{j}-1}$. We apply the diffeomorphism $\chi$ and obtain that the vectors:

$$
\chi_{*}\left[\widehat{X}_{i_{1}^{1}},\left[\ldots, \widehat{X}_{i_{m_{1}}^{1}}\right] \ldots\right](x), \ldots, \chi_{*}\left[\widehat{X}_{i_{1}^{n}},\left[\ldots, \widehat{X}_{i_{m_{n}}^{n}}\right] \ldots\right](x),
$$

form a basis of $\mathbb{R}^{n}$ for any $x$ from a neighborhood of 0 . Moreover, the vectors (6) form a basis of $\mathbb{R}^{n}$ for any $x \in \mathbb{R}^{n}$ thanks to the $\nu$-homogeneity of $\chi_{*} \widehat{X}_{i}$.

Take a compact $K \subset \mathbb{R}^{n}$. There exists $\varepsilon_{K}>0$ such that the vectors:

$$
\left[Z_{i_{1}^{1}}^{\varepsilon},\left[\ldots, Z_{i_{m_{1}}^{1}}^{\varepsilon}\right] \ldots\right](x), \ldots,\left[Z_{i_{1}^{n}}^{\varepsilon},\left[\ldots, Z_{i_{m_{n}}^{n}}^{\varepsilon}\right] \ldots\right](x),
$$

form a basis of $\mathbb{R}^{n}$ for any $(x, \varepsilon) \in D_{K}=\left\{(x, \varepsilon)|x \in K,| \varepsilon \mid \leqslant \varepsilon_{K}\right\}$.

Finally, we define a family of controls $w_{\bar{s}}, \bar{s}=\left(s_{1}, \ldots, s_{n}\right), s_{j} \in \mathbb{R}, j=1, \ldots, n$, by the rule:

$$
w_{\bar{s}}=\left\{\begin{array}{cc}
n \varsigma\left[i_{1}^{1} \ldots i_{m_{1}}^{1}\right]_{s_{1}}\left(\frac{t}{n}\right), & 0 \leqslant t<\frac{1}{n}, \\
\ldots \ldots \ldots \ldots \ldots \ldots & \ldots \ldots \ldots \ldots \\
n_{\zeta}\left[i_{1}^{n} \ldots i_{m_{n}}^{n}\right]_{s_{n}}\left(\frac{t}{n}\right), & \frac{n-1}{n} \leqslant t \leqslant 1 .
\end{array}\right.
$$


Let the mapping $\mathfrak{Z}_{u}^{\varepsilon}$ be defined similarly to $\mathfrak{Z}_{u}$, replacing the field $Z_{i}$ by the field $Z_{i}^{\varepsilon}$. Then:

$$
\left.\frac{\partial}{\partial s_{j}} \mathfrak{Z}_{w_{\bar{s}}}^{\varepsilon}\right|_{\bar{s}=0}=\left[Z_{i_{1}^{j}}^{\varepsilon},\left[\ldots, Z_{i_{m_{j}}^{j}}^{\varepsilon}\right] \ldots\right] .
$$

In particular, the mapping $\Phi_{x}^{\varepsilon}: \bar{s} \mapsto\left(\mathfrak{Z}_{w_{\bar{s}}}^{\varepsilon}(x)-x\right)$ is a submersion at 0 for any $x \in K$, $|\varepsilon| \leqslant \varepsilon_{K} ; \Phi_{x}^{\varepsilon}(0)=0$.

Recall that the family of mappings $\Phi_{x}^{\varepsilon}$ is smooth with respect to the parameters $(\varepsilon, x)$, and $(\varepsilon, x)$ belongs to the compact set $D_{K}$. Hence the inverse mapping $\left(\Phi_{x}^{\varepsilon}\right)^{-1}$ is well defined on a ball $\left\{z \in \mathbb{R}^{n}:|z| \leqslant \delta\right\}$, the radius $\delta$ of which does not depend on $(x, \varepsilon)$. Clearly, $\left(\Phi_{x}^{\varepsilon}\right)^{-1}(z) \leqslant c^{\prime}|z|$ for some constant $c^{\prime}$. Hence the equation $\mathfrak{Z}_{w_{\bar{s}}}^{\varepsilon}(x)=y$ has a solution $\bar{s}$ such that $|s| \leqslant c^{\prime}|x-y|$ if $x \in K,|x-y| \leqslant \delta$, and $|\varepsilon| \leqslant \varepsilon_{K}$. It follows that $d_{\varepsilon}(x, y) \leqslant\left\|w_{\bar{s}}\right\| \leqslant c^{\prime \prime}|s|^{1 / 2^{l}} \leqslant c|x-y|^{1 / 2^{l}}$.

THEOREM 3. $-\rho_{\varepsilon} \rightarrow \hat{\rho}$ uniformly on compact subsets of $\mathbb{R}^{n}$ as $\varepsilon \rightarrow 0$.

Proof. - Thanks to the equicontinuity of the family of functions $\left.\rho_{\varepsilon}\right|_{K}$ (Lemma 1) it is enough to prove the pointwise convergence $\rho_{\varepsilon} \rightarrow \hat{\rho}$ as $\varepsilon \rightarrow 0$.

Take $x \in \mathbb{R}^{n}$; there exists $\hat{u} \in U_{\hat{\rho}(x)}$ such that $\hat{f}(\hat{u})=x$. Let $x_{\varepsilon}=f_{\varepsilon}(\hat{u})$. We have $\rho_{\varepsilon}\left(x_{\varepsilon}\right) \leqslant\|\hat{u}\|=\hat{\rho}(x)$. Hence:

$$
\rho_{\varepsilon}(x)=\rho_{\varepsilon}\left(x_{\varepsilon}\right)+\rho_{\varepsilon}(x)-\rho_{\varepsilon}\left(x_{\varepsilon}\right) \leqslant \hat{\rho}(x)+\left|\rho_{\varepsilon}(x)-\rho_{\varepsilon}\left(x_{\varepsilon}\right)\right| .
$$

According to Theorem 2, $x_{\varepsilon} \rightarrow x$ as $\varepsilon \rightarrow 0$. Now Lemma 1 implies the inequality $\lim \sup _{\varepsilon \rightarrow 0} \rho_{\varepsilon}(x) \leqslant \hat{\rho}(x)$.

For any $\varepsilon$ small enough, there exists $u_{\varepsilon} \in U_{\rho_{\varepsilon}(x)}$ such that $f_{\varepsilon}\left(u_{\varepsilon}\right)=x$. The equicontinuity of $\rho_{\varepsilon}$ and the identity $\rho_{\varepsilon}(0)=0$ imply that $\left\|u_{\varepsilon}\right\|=\rho_{\varepsilon}(x)$ are uniformly bounded. Let $\hat{x}_{\varepsilon}=\hat{f}\left(u_{\varepsilon}\right)$. We have $\hat{\rho}\left(\hat{x}_{\varepsilon}\right) \leqslant \rho_{\varepsilon}(x)$. Hence:

$$
\hat{\rho}(x)=\hat{\rho}\left(\hat{x}_{\varepsilon}\right)-\hat{\rho}\left(\hat{x}_{\varepsilon}\right)+\hat{\rho}(x) \leqslant \rho_{\varepsilon}(x)+\left|\hat{\rho}\left(\hat{x}_{\varepsilon}\right)-\hat{\rho}(x)\right| .
$$

It follows from Theorem 2 that $\hat{x}_{\varepsilon} \rightarrow x$ as $\varepsilon \rightarrow 0$. The continuity of $\hat{\rho}$ implies the inequality $\hat{\rho}(x) \leqslant \liminf _{\varepsilon \rightarrow 0} \rho_{\varepsilon}(x)$.

Finally, $\lim _{\varepsilon \rightarrow 0} \rho_{\varepsilon}(x)=\hat{\rho}(x)$.

The following proposition is a modification of a result by Jacquet [17].

PROPOSITION 3. - Let $\mathcal{M}_{r}=\left\{u \in U_{r}: \exists \alpha \in(0,1]\right.$ s.t. $\alpha u$ is minimal for (2) $\}$. Then $\overline{\mathcal{M}}_{r}$ is a compact subset of the Hilbert sphere $U_{r}$ and $\hat{f}\left(\overline{\mathcal{M}}_{r} \backslash \mathcal{M}_{r}\right) \subset \hat{\rho}^{-1}(r)$; in particular, any element of $\overline{\mathcal{M}}_{r} \backslash \mathcal{M}_{r}$ is a minimal control for system $(\hat{2})$.

Proof. - First of all, the mappings $f$ and $\hat{f}$ are weakly continuous; this is a standard fact, see [1] for a few lines proof. Let $v_{n} \in \mathcal{M}_{r}, n=1,2, \ldots$, be a weakly convergent sequence in $L_{2}^{k}[0,1]$, such that $\alpha_{n} v_{n}$ are minimal. Let $v$ be the weak limit of $v_{n}$, $\|v\| \leqslant r$. We may assume without lack of generality that $\exists \lim _{n \rightarrow \infty} \alpha_{n}=\alpha$. There are two possibilities.

(1) $\alpha>0$. We have $\alpha r=\lim _{n \rightarrow \infty} \alpha_{n} r=\lim _{n \rightarrow \infty} \rho\left(f\left(\alpha_{n} v_{n}\right)\right)=\rho(f(\alpha v))$. Hence the length of the trajectory associated to the control $\alpha v$ is $\alpha r$. In particular, $\|\alpha v\| \geqslant \alpha r$. We 
already know that $\|v\| \leqslant r$. Thus $\|v\|=r, v$ is normalized and belongs to $\mathcal{M}_{r}$. Moreover, the sequence $v_{n}$ is strongly convergent since the weak and strong topologies coincide on the Hilbert sphere.

(2) $\alpha=0$. We have $\hat{\rho}(\hat{f}(v))=\lim _{n \rightarrow \infty} \hat{\rho}\left(\hat{f}\left(v_{n}\right)\right)$. Theorems 2, 3, and Lemma 1 make it possible to replace $\hat{\rho}$ by $\rho_{\alpha_{n}}$ and $\hat{f}$ by $f_{\alpha_{n}}$ in the right-hand side of the last equality. We obtain

$$
\hat{\rho}(\hat{f}(v))=\lim _{n \rightarrow \infty} \rho_{\alpha_{n}}\left(f_{\alpha_{n}}\left(v_{n}\right)\right)=\lim _{n \rightarrow \infty} \frac{1}{\alpha_{n}} \rho\left(f\left(\alpha_{n} v_{n}\right)\right)=\lim _{n \rightarrow \infty} r=r .
$$

Now the same arguments as in the case (1) show that $v$ is normalized and $\|v\|=1$.

\section{Subanalyticity and nilpotentization}

In this section we assume that the Riemannian manifold $M$ and the distribution $\Delta$ are real analytic. Then we can assume (and we do so) that the vector fields $X_{1}, \ldots, X_{k}$ and the adapted coordinate mapping are real analytic.

THEOREM 4. - If the germ of $\rho$ at $q_{0}$ is subanalytic, then $\hat{\rho}$ is subanalytic.

Proof. - Let $S^{n-1}$ be the unit sphere in $\mathbb{R}^{n}$ and let $\varepsilon>0$ be such that $\rho\left(\chi^{-1}\left(\delta_{t} x\right)\right)$ is well defined for all $x \in S^{n-1},|t| \leqslant \varepsilon$. Then $(t, x) \mapsto \rho\left(\chi^{-1}\left(\delta_{t} x\right)\right)$ is a subanalytic function on the product $(-\varepsilon, \varepsilon) \times S^{n-1}$. Moreover,

$$
\hat{\rho}(x)=\lim _{t \rightarrow 0} \rho_{t}(x)=\lim _{t \rightarrow 0} \frac{1}{t} \rho\left(\chi^{-1}\left(\delta_{t} x\right)\right) .
$$

Hence $\hat{\rho}$ is a subanalytic function on the compact algebraic manifold $S^{n-1}$ (see $[13,16]$ ). Now the quasi-homogeneity of $\hat{\rho}, \hat{\rho}\left(\delta_{t} x\right)=|t| \hat{\rho}(x)$, implies the subanalyticity of $\hat{\rho}$ on the whole $\mathbb{R}^{n}$.

So the subanalyticity of $\rho$ implies the same property for $\hat{\rho}$. It is hard to expect that the inverse implication is always true. We are going however to show that it is true very often. Namely, $\rho$ is subanalytic if the nilpotentization $(\hat{2})$ of the original system satisfies general sufficient conditions for subanalyticity of sub-Riemannian balls developed in [1]. We point out that, in general, the subanalyticity of all balls $\rho^{-1}([0, r])$ (i.e. the Lebesgue sets of $\rho$ ) does not imply at all the subanalyticity of $\rho$ (i.e. the graph of $\rho$ ); see the next section to appreciate a sharp difference between these two kinds of subanalyticity. At the same time, the subanalyticity of the balls $\hat{\rho}^{-1}([0, \varepsilon])$ is equivalent to the subanalyticity of $\hat{\rho}$ itself, by the quasi-homogeneity of $\hat{\rho}$.

Let us recall the background on sub-Riemannian geodesics we need to formulate the abovementioned subanalyticity conditions. First we set $f_{r}=\left.f\right|_{U_{r}}$, the restriction of the endpoint mapping to the Hilbert sphere. The critical points of the mapping $f_{r}: U_{r} \rightarrow M$ are called extremal controls and the corresponding solutions of Eq. (2) are called extremal trajectories or sub-Riemannian geodesics. It is easy to check that all minimal controls are extremal ones. The geodesics associated to minimal controls are also called minimal. 
An extremal control $u$ and the corresponding geodesic $q(\cdot)$ are regular if $u$ is a regular point of $f$; otherwise they are singular or abnormal.

Let $D_{u} f: L_{2}^{k}[0,1] \rightarrow T_{f(u)} M$ be the differential of $f$ at $u$. Extremal controls (and only them) satisfy the equation:

$$
\lambda D_{u} f=v u
$$

with some "Lagrange multipliers" $\lambda \in T_{f(u)}^{*} M \backslash 0, v \in \mathbb{R}$. Here $\lambda D_{u} f$ is the composition of the linear mapping $D_{u} f$ and the linear form $\lambda: T_{f(u)} M \rightarrow \mathbb{R}$, i.e. $\left(\lambda D_{u} f\right) \in$ $L_{2}^{k}[0,1]^{*}=L_{2}^{k}[0,1]$. We have $v \neq 0$ for regular extremal controls, while for abnormal controls $v$ can be taken 0 . In principle, abnormal controls may admit Lagrange multipliers with both zero and nonzero $v$. If it is not the case, then the control and the geodesic are called strictly abnormal.

Pontryagin's maximum principle gives an efficient way to solve Eq. (7), i.e. to find extremal controls and Lagrange multipliers. A coordinate free formulation of the maximum principle uses the canonical symplectic structure on the cotangent bundle $T^{*} M$. The symplectic structure associates a Hamiltonian vector field $\vec{a} \in$ Vec $T^{*} M$ to any smooth function $a: T^{*} M \rightarrow \mathbb{R}$.

We define the functions $h_{i}, i=1, \ldots, k$, and $h$ on $T^{*} M$ by the formulas

$$
h_{i}(\psi)=\left\langle\psi, X_{i}(q)\right\rangle, \quad h(\psi)=\frac{1}{2} \sum_{i=1}^{k} h_{i}^{2}(\psi), \quad \forall q \in M, \psi \in T_{q}^{*} M
$$

Pontryagin's maximum principle implies the following:

Proposition 4. $-A$ triple $(u, \lambda, v)$ satisfies $E q$. (7) if and only if there exists a solution $\psi(t), 0 \leqslant t \leqslant 1$, to the system of differential and pointwise equations:

$$
\dot{\psi}=\sum_{i=1}^{k} u_{i}(t) \vec{h}_{i}(\psi), \quad h_{i}(\psi(t))=v u_{i}(t)
$$

with boundary conditions $\psi(0) \in T_{q_{0}}^{*} M, \psi(1)=\lambda$.

Here $(\psi(t), v)$ are Lagrange multipliers for the extremal control $u_{t}: \tau \mapsto t u(t \tau)$; in other words, $\psi(t) D_{u_{t}} f=v u_{t}$.

Note that abnormal geodesics are still geodesics after an arbitrary reparametrization, while regular geodesics are automatically normalized. We say that a geodesic is quasiregular if it is normalized and is not strictly abnormal. Setting $v=1$ we obtain a simple description of all quasi-regular geodesics.

COROLLARY 1.-Quasi-regular geodesics are exactly projections on $M$ of the solutions of the differential equation $\dot{\psi}=\vec{h}(\psi)$ with initial conditions $\psi(0) \in T_{q_{0}}^{*} M$. If $h(\psi(0))$ is small enough, then such a solution exists (i.e. is defined on the whole segment $[0,1])$. The length of the geodesic is equal to $\sqrt{2 h(\psi(0))}$ and the Lagrange multiplier $\lambda=\psi(1)$.

Corollary 1 provides a parametrization of the space of quasi-regular geodesics by the points of an open subset $\Psi$ of $T_{q_{0}}^{*} M$. Namely, $\Psi$ consists of $\psi_{0} \in T_{q_{0}}^{*} M$ such that 
the solution $\psi(t)$ to the equation $\dot{\psi}=\vec{h}(\psi)$ with the initial condition $\psi(0)=\psi_{0}$ is defined for all $t \in[0,1]$. The space of quasi-regular geodesics of a prescribed length $r$, small enough, are parametrized by the points of the manifold $h^{-1}\left(\frac{r^{2}}{2}\right) \cap T_{q_{0}}^{*} M \subset \Psi$. This manifold is diffeomorphic to $\mathbb{R}^{n-k} \times S^{k-1}$. The composition of the given parametrization with the endpoint mapping $f$ is the exponential mapping $\mathcal{E}: \Psi \rightarrow M$. Thus $\mathcal{E}(\psi(0))=$ $\pi(\psi(1))$, where $\pi: T^{*} M \rightarrow M$ is the canonical projection.

Throughout the paper the "hat" over a symbol means that we replace the original system (2) by its nilpotentization $(\hat{2})$ in the construction of the object denoted by the symbol. In particular, $\hat{h}$ is the Hamiltonian and $\hat{\mathcal{E}}$ is the exponential mapping for the system $(\hat{2})$. Besides that, we denote by $h^{\varepsilon}$ and $\mathcal{E}^{\varepsilon}$ the Hamiltonian and the exponential mapping for the system:

$$
\dot{x}=\sum_{i=1}^{k} u_{i} Z_{i}^{\varepsilon}(x), \quad x \in \mathbb{R}^{n},
$$

where $Z_{i}^{\varepsilon}=\varepsilon \delta_{\varepsilon^{*}}^{-1} \chi_{*} X_{i}$. Recall that system $\left(2^{\varepsilon}\right)$ produces the endpoint mapping $f_{\varepsilon}$ and sub-Riemannian distance $\rho_{\varepsilon}$. Note that $(\varepsilon, x) \mapsto Z_{i}^{\varepsilon}(x)$ are real analytic vector functions and $Z_{i}^{0}=\widehat{X}_{i}$. Hence $h^{\varepsilon}(\psi), \mathcal{E}^{\varepsilon}(\psi)$ are also analytic with respect to $(\varepsilon, \psi)$ and $h^{0}=\hat{h}$, $\mathcal{E}^{0}=\hat{\mathcal{E}}$.

Our results on subanalyticity of the distance function $\rho$ are based upon the following statement.

PROPOSITION 5. - Assume that there exists a compact $K \subset T_{q_{0}}^{*} M$ such that $\rho_{r}^{-1}(1) \subset$ $\mathcal{E}\left(K \cap\left(h^{r}\right)^{-1}\left(\frac{1}{2}\right)\right)$ for any small enough nonnegative $r$. Then the germ of $\rho$ at $q_{0}$ is subanalytic.

Proof. - We have:

$$
\rho(q)=\min \left\{r: \exists \psi \in K, \text { such that } h^{r}(\psi)=\frac{1}{2}, \delta_{r} \mathcal{E}^{r}(\psi)=\chi(q)\right\},
$$

for any $q$ in a neighborhood of $q_{0}$. One can enlarge the compact $K$, if necessary, to make it semi-analytic. The subanalyticity of $\rho$ follows now from [23, Proposition 1.3.7], thanks to the analyticity of $\mathcal{E}^{r}(\psi)$ and $h^{r}(\psi)$ with respect to $(r, \psi)$.

Let $u \in U_{r}$ be an extremal control, i.e. a critical point of $f_{r}$. The Hessian of $f_{r}$ at $u$ is a quadratic mapping

$$
\operatorname{Hes}_{u} f_{r}: \operatorname{ker} D_{u} f_{r} \rightarrow \operatorname{coker} D_{u} f_{r} .
$$

This is a coordinate free part of the second derivative of $f_{r}$ at $u$. Let $(\lambda, v)$ be Lagrange multipliers associated with $u$ so that Eq. (7) is satisfied. Then the covector $\lambda: T_{f(u)} M \rightarrow$ $\mathbb{R}$ annihilates $\operatorname{im} D_{u} f_{r}$ and the composition:

$$
\lambda \operatorname{Hes}_{u} f_{r}: \operatorname{ker} D_{u} f_{r} \rightarrow \mathbb{R},
$$

is well-defined. 
The quadratic form (9) is the second variation of the sub-Riemannian problem at $(u, \lambda, \nu)$. We have:

$$
\lambda \operatorname{Hes}_{u} f_{r}(v)=\lambda D_{u}^{2} f(v, v)-v|v|^{2}, \quad v \in \operatorname{ker} D_{u} f_{r} .
$$

Let $q(\cdot)$ be the geodesic associated with the control $u$. We set:

$$
\operatorname{ind}(f ; u, \lambda, v)=\operatorname{ind}_{+}\left(\lambda \operatorname{Hes}_{u} f_{r}\right)-\operatorname{dim} \operatorname{coker} D_{u} f_{r},
$$

where $\operatorname{ind}_{+}\left(\lambda \operatorname{Hes}_{u} f_{r}\right)$ is the positive inertia index of the quadratic form $\lambda \operatorname{Hes}_{u} f_{r}$. Decoding some of the symbols we can rewrite:

$$
\begin{aligned}
\operatorname{ind}(f ; u, \lambda, v)= & \sup \left\{\operatorname{dim} V: V \subset \operatorname{ker} D_{u} f_{r}, \lambda D_{u}^{2} f(v, v)>v|v|^{2}, \forall v \in V \backslash 0\right\} \\
& -\operatorname{dim}\left\{\lambda^{\prime} \in T_{f(u)}^{*} M: \lambda^{\prime} D_{u} f_{r}=0\right\} .
\end{aligned}
$$

The value of ind $(f ; u, \lambda, v)$ may be an integer or $+\infty$.

Remark. - The index (10) does not depend on the choice of the orthonormal frame $X_{1}, \ldots, X_{k}$ and is actually a characteristic of the geodesic $q(\cdot)$ and the Lagrange multipliers $(\lambda, \nu)$. Indeed, a change of the frame leads to a smooth transformation of the Hilbert manifold $U_{r}$ and to a linear transformation of variables in the quadratic form $\lambda \operatorname{Hes}_{u} f_{r}$ and the linear mapping $D_{u} f_{r}$. Both terms in the right-hand side of (10) remain unchanged.

The next theorem presents the most important properties of index (10); see [1,5] and references there for proofs and details.

THEOREM 5.-(1) The integer-valued function $(f, u, \lambda, v) \mapsto \operatorname{ind}(f ; u, \lambda, v)$ is lower semicontinuous for the $C^{2}$ topology in the space of the mappings $f: L_{2}^{k}[0,1] \rightarrow M$.

(2) For any minimal control $u$ there exist Lagrange multipliers $\lambda, v$ such that $\operatorname{ind}(f ; u, \lambda, v)<0$.

Now we are ready to formulate the main result of this section. It is a generalization of some results from $[1,17]$.

THEOREM 6. - Assume that ind $(\hat{f} ; \hat{u}, \hat{\lambda}, 0) \geqslant 0$ for any nonzero abnormal control $\hat{u}$ of the nilpotent system $(\hat{2})$ and any associated Lagrange multipliers $(\hat{\lambda}, 0)$. Then the germ of $\rho$ at $q_{0}$ is subanalytic.

Proof. - First we'll prove that no sufficiently small strictly abnormal control of the original system (2) is minimal.

Assume on the contrary that $u_{m}, m=1,2, \ldots$, is a sequence of minimal strictly abnormal controls, $\left\|u_{m}\right\|=\varepsilon_{m}, \varepsilon_{m} \rightarrow 0(m \rightarrow \infty)$. The minimality of $u_{m}$ implies the existence of a nonzero $\lambda_{m} \in T_{f\left(u_{m}\right)}^{*} M$ such that:

$$
\lambda_{m} D_{u_{m}} f=0, \quad \operatorname{ind}\left(f ; u_{m}, \lambda_{m}, 0\right)<0 .
$$

Set $v_{m}=\frac{1}{\varepsilon_{m}} u_{m}, \mu_{m}=\delta_{\varepsilon_{m}}^{*} \lambda_{m}$ and rewrite relations (11) in the form:

$$
\mu_{m} D_{v_{m}} f_{\varepsilon_{m}}=0, \quad \operatorname{ind}\left(f_{\varepsilon_{m}} ; v_{m}, \mu_{m}, 0\right)<0 .
$$


According to Proposition 3, we may assume that there exists a (strong) $\lim _{m \rightarrow \infty} v_{m}=v$. Of course, we may also assume that there exists $\lim _{m \rightarrow \infty} \mu_{m}=\mu \neq 0$. Theorem 2 implies that $\mu D_{v} \hat{f}=0$, i.e. $v$ is an abnormal control for the nilpotent system $(\hat{2})$. On the other hand, the lower semicontinuity of ind implies that ind $(\hat{f} ; v, \mu, 0)<0$ and we come to a contradiction.

Therefore, any short enough minimal geodesic is quasi-regular. Hence:

$$
\rho(q)=\min \left\{r: \exists \psi \in T_{0}^{*} \mathbb{R}^{n} \text {, such that } h^{r}(\psi)=\frac{1}{2}, \delta_{r} \mathcal{E}^{r}(\psi)=\chi(q)\right\} .
$$

Now it remains only to show that, in relation (12), $T_{0}^{*} \mathbb{R}^{n}$ can be replaced by a compact subset $K \subset T_{0}^{*} \mathbb{R}^{n}$ and to apply Proposition 5 .

Denote by $u_{\psi(0)}^{r}$ the extremal control associated with $\psi(0) \in\left(h^{r}\right)^{-1}\left(\frac{1}{2}\right)$ so that $\mathcal{E}^{r}(\psi(0))=f_{r}\left(u_{\psi(0)}\right)$. We have $u_{\psi(0)}^{r}=\left(h_{1}^{r}(\psi(\cdot)), \ldots, h_{k}^{r}(\psi(\cdot))\right)$ (see Proposition 4 and its corollary). In particular, $u_{\psi(0)}^{r}$ depends continuously on $\psi(0)$. We set:

$$
\begin{gathered}
K_{r}=\left\{\psi(0) \in\left(h^{r}\right)^{-1}\left(\frac{1}{2}\right): u_{\psi(0)}^{r} \text { is minimal for }\left(2^{r}\right), \operatorname{ind}\left(f_{r} ; u_{\psi(0)}^{r}, \psi(1), 0\right)<0\right\}, \\
K^{\varepsilon}=\bigcup_{0 \leqslant r \leqslant \varepsilon} K_{r} .
\end{gathered}
$$

It follows from Theorem 5 that one can replace $T_{0}^{*} \mathbb{R}^{n}$ by $K^{\varepsilon}$ in (12) if $q$ lies in $\rho^{-1}([0, \varepsilon])$. We have shown above that the system

$$
\mu D_{v} f_{\varepsilon}=0, \quad \operatorname{ind}\left(f_{\varepsilon} ; v, \mu, 0\right)<0, \quad \mu \in \mathbb{R}^{n} \backslash 0, v \in U_{1},
$$

has no solutions for $\varepsilon$ small enough, and we assume $\varepsilon$ to be so small. We are going to prove that $K^{\varepsilon}$ is compact.

Take a sequence $\psi_{m}(0) \in K_{r_{m}} \subset K^{\varepsilon}, m=1,2, \ldots$ We have to find a convergent subsequence. $K_{0}$ is compact in virtue of [1, Theorem 5] applied to system $(\hat{2})$. Hence we may assume that $r_{m}>0$ for all $m$. Moreover, we may assume that there exists $\lim _{m \rightarrow \infty} r_{m}=\bar{r}$. The controls $u_{\psi_{m}(0)}^{r_{m}}$ belong to $\mathcal{M}_{\varepsilon}$; according to Proposition 3, there exists a convergent subsequence of this sequence of controls and its limit is minimal for system $\left(2^{\bar{r}}\right)$. To simplify notations, we assume that the sequence $u_{\psi_{m}(0)}^{r_{m}}, m=1,2, \ldots$, is already convergent and $\lim _{m \rightarrow \infty} u_{\psi_{m}(0)}^{r_{m}}=\bar{u}$.

It follows from Proposition 4 that $\psi_{m}(1) D_{u_{\psi_{m}(0)}^{r_{m}}} f_{r_{m}}=u_{\psi_{m}(0)}^{r_{m}}$. There are two possibilities: either $\left|\psi_{m}(1)\right| \rightarrow \infty(m \rightarrow \infty)$ or $\psi_{m}(1), m=1,2, \ldots$, contains a convergent subsequence.

In the first case we come to the equation $\bar{\mu} D_{\bar{u}} f_{\bar{r}}=0$, where $\bar{\mu}$ is a limiting point of the sequence $\frac{1}{\left|\psi_{m}(1)\right|} \psi_{m}(1),|\bar{\mu}|=1$. The lower semicontinuity of ind implies the inequality $\operatorname{ind}\left(f_{\bar{r}} ; \bar{u}, \bar{\mu}, 0\right)<0$. We come to a contradiction with our assumption on $\varepsilon$ since $\bar{r} \leqslant \varepsilon$.

In the second case let $\psi_{m_{l}}(1), l=1,2, \ldots$, be a convergent subsequence. Then $\psi_{m_{l}}(0), l=1,2, \ldots$, is also convergent, $\exists \lim _{l \rightarrow \infty} \psi_{m_{l}}(0)=\bar{\psi}(0)$. Then $\bar{u}=u_{\bar{\psi}(0)}^{\bar{r}}$ and $\operatorname{ind}\left(f_{\bar{r}} ; \bar{u}, \bar{\psi}(1), 1\right)<0$ because of the lower semicontinuity of ind. Hence $\bar{\psi}(0) \in K_{\bar{r}} \subset$ $K^{\varepsilon}$ and we are done. 
To apply the last theorem we need a way to evaluate our index. There is a well developed theory about that, see [1] for some references. In the next proposition we formulate just the most simple and easy to check necessary conditions for the finiteness of the ind. A detailed proof can be found in [4, Appendix 2].

Proposition 6. - Assume that $u(\cdot)$ is an abnormal control and $\psi(\cdot) \neq 0$ satisfies (8) for $v=0$. If ind $(f ; u(\cdot), \psi(1), 0)<\infty$, then:

$$
\begin{gathered}
\left\{h_{i}, h_{j}\right\}(\psi(t))=0 \quad \forall i, j \in\{1, \ldots, k\}, \\
\sum_{i, j=1}^{k}\left\{h_{i},\left\{h_{j}, \sum_{i=1}^{k} u_{l}(t) h_{l}\right\}\right\} v_{i} v_{j} \leqslant 0 \quad \forall\left(v_{1}, \ldots, v_{k}\right) \in \mathbb{R}^{k},
\end{gathered}
$$

for almost all $t \in[0,1]$, where $\{a, b\}=\vec{a} b$ is the Poisson bracket of the Hamiltonians $a, b$.

Remark. - Identity (13) is called the Goh condition while inequality (14) is the generalized Legendre condition. It is easy to see that both conditions are actually intrinsic: Identity (13) does not depend on the choice of the orthonormal frame $X_{1}, \ldots, X_{k}$ since $h_{i}(\psi(t)), i=1, \ldots, k$, vanish anyway. Inequality (14) does not depend on the choice of the orthonormal frame provided that (13) is satisfied.

We say that $u(\cdot)$ is a Goh control if (13) is satisfied for an appropriate $\psi(\cdot)$; it is a Goh-Legendre control if both (13) and (14) are satisfied.

COROLLARY 2. - If the nilpotent system (2) does not admit nonzero Goh-Legendre abnormal controls, then the germ of $\rho$ at $q_{0}$ is subanalytic.

The system (2) is said to be medium fat if:

$$
T_{q_{0}} M=\Delta_{q_{0}}^{2}+\operatorname{span}\left\{\left[X,\left[X_{i}, X_{j}\right]\right]\left(q_{0}\right): i, j=1, \ldots, k\right\}
$$

for any $X \in \bar{\Delta}, X\left(q_{0}\right) \neq 0$ (see [5]). Medium fat systems do not admit nontrivial Goh controls. It follows directly from the definitions that a system is medium fat if and only if its nilpotentization is. We come to the following:

COROLlary 3. - If the system (2) is medium fat, then the germ of $\rho$ at $q_{0}$ is subanalytic.

It is proved in [5] that generic germs of distributions are medium fat for $n \leqslant(k-$ $1) k+1$. This gives the following general result.

THEOREM 7. - Assume that $n \leqslant(k-1) k+1$. Then the germ of the sub-Riemannian distance function associated with a generic germ of a rank $k$ distribution on an $n$-dimensional real-analytic Riemannian manifold is subanalytic.

\section{Exclusivity of Goh controls for rank $>2$ distributions}

First we'll make precise the term exclusivity. Rank $k$ distributions on $M$ are smooth sections of the "Grassmannization" $H_{k} T M$ of the tangent bundle $T M$. The space of 
sections is endowed with the $C^{\infty}$ Whitney topology and is denoted by $\overline{H_{k} T M}$. Smooth families of distributions parametrized by the finite dimensional manifold $N$ are sections of the bundle $p_{*}^{N} H_{k} T M$ over $N \times M$ induced by the standard projection $p^{N}: N \times M \rightarrow$ $M$. Let $\mathcal{A} \subset \overline{H_{k} T M}$ be a set of distributions. We say that $\mathcal{A}$ has codimension $\infty$ in $\overline{H_{k} T M}$ if the subset:

$$
\left\{D \in \overline{p_{*}^{N} H_{k} T M}:\left.D\right|_{x \times M} \notin \mathcal{A}, \forall x \in N\right\},
$$

is everywhere dense in $\overline{p_{*}^{N} H_{k} T M}, \forall N$.

We will also use a real-analytic version of the definition, just given. The only difference with the smooth case is that the manifolds and the sections are assumed to be real-analytic, while the topology remains the same Whitney topology.

THEOREM 8. - For any $k \geqslant 3$, the distributions admitting nonzero Goh controls form a subset of codimension $\infty$ in the space of all smooth rank $k$ distributions on $M$.

Proof. - We start with a weaker result related to smooth Goh controls. Namely, we are going to prove that the distributions that admit nonzero $C^{\infty}$ Goh controls form a subset of codimension $\infty$ in the space of rank $k \geqslant 3$ distributions. Thom transversality theorem allows to reduce the proof to calculations in the jet spaces. Let $\mathcal{J}^{m}(n, k)$ be the space of $m$-jets at 0 of $k$-tuples of vector fields in $\mathbb{R}^{n}$ and $\mathcal{J}_{o}^{m}(n, k)=\left\{\left(X_{1}, \ldots, X_{k}\right) \in\right.$ $\left.\mathcal{J}^{m}(n, k): X_{1}(0) \wedge \cdots \wedge X_{k}(0) \neq 0\right\}$ be the space of $m$-jets of $k$-frames. To any vector field $X_{i}$ we associate the Hamiltonian $h_{i}(\xi, x)=\left\langle\xi, X_{i}(x)\right\rangle,(\xi, x) \in \mathbb{R}^{n *} \times \mathbb{R}^{n}$ and the Hamiltonian field $\vec{h}_{i}(\xi, x)=\sum_{j=1}^{n}\left(\frac{\partial h_{i}}{\partial \xi^{j}} \frac{\partial}{\partial x^{j}}-\frac{\partial h_{i}}{\partial x^{j}} \frac{\partial}{\partial \xi^{j}}\right)$. Set $\psi=(\xi, x)$; the Goh controls for the system $\dot{x}=\sum_{i=1}^{k} u_{i}(t) X_{i}(x), x(0)=0$, are admissible controls $u=$ $\left(u_{1}(\cdot), \ldots, u_{k}(\cdot)\right)$ such that there exist:

$$
\begin{gathered}
\psi(\cdot)=(\xi(\cdot), x(\cdot)), \quad \xi(0) \neq 0, \quad x(0)=0, \quad \dot{\psi}=\sum_{i=1}^{k} u_{i}(t) \vec{h}_{i}(\psi), \\
h_{i}(\psi(t))=\left\{h_{i}, h_{j}\right\}(\psi(t)) \equiv 0, \quad i, j=1, \ldots, k .
\end{gathered}
$$

Working in the jet space we try to solve Eqs. (16) not precisely but up to a certain order. We say that the $m$-jet of $\left(X_{1}, \ldots, X_{k}\right)$ is Goh-compatible if there exists a nontrivial smooth solution $(u, \psi(\cdot))$ of (15) such that the functions $t \mapsto h_{i}(\psi(t)), t \mapsto$ $\left\{h_{i}, h_{j}\right\}(\psi(t)), i, j=1, \ldots, k$, have zero $m$-jets at $t=0$.

Let $\mathcal{A}^{m} \subset \mathcal{J}_{o}^{m}(n, k)$ be the set of all Goh-compatible $m$-jets. Standard transversality techniques reduce the expected result about the set of distributions admitting nontrivial $C^{\infty}$ Goh controls to the following lemma.

LEMmA 2. $-\mathcal{A}^{m}$ is an algebraic subset of the linear space $\mathcal{J}_{o}^{m}(n, k)$ and $\operatorname{codim} \mathcal{A}^{m} \rightarrow \infty$ as $m \rightarrow \infty$.

Proof. - Differentiating (16) $m$ times in virtue of (15) at $t=0$ leads to a system of polynomial equations on $\xi(0), u_{i}(0), \ldots, u_{i}^{(m-1)}(0), i=1, \ldots, k$. Actually, these equations are even linear with respect to $\xi(0)$. The set $\mathcal{A}^{m}$ is thus automatically algebraic. 
Any reparametrization of a Goh trajectory is still Goh. In particular, we may normalize one of the coordinates of the nontrivial smooth Goh control assuming that $u_{i_{0}} \equiv 1$ for some $i_{0}$. Without lack of generality, we may compute everything only in the case $i_{0}=1$. Moreover, any nonvanishing vector field is locally rectifiable and gauge transformations $X_{1} \mapsto X_{1}, X_{i} \mapsto X_{i}(x)+a_{i}(x) X_{1}(x), i=2, \ldots, k$, do not change Gohcompatibility.

Hence we may assume that:

$$
X_{1}=\frac{\partial}{\partial x^{1}}, \quad X_{i}(x)=\sum_{j=2}^{n} a_{i j}(x) \frac{\partial}{\partial x^{j}}, \quad i=2, \ldots, k,
$$

where $a_{i j}(x)$ are polynomials of degree $m$. In particular, $X_{i}=\sum_{\alpha=0}^{m}\left(x^{1}\right)^{\alpha} Y_{i}^{\alpha}(y)$, where $y=\left(x^{2}, \ldots, x^{n}\right),\left(Y_{2}^{\alpha}, \ldots, Y_{k}^{\alpha}\right) \in \mathcal{J}^{m}(n-1, k-1), \alpha=1, \ldots, m$, and $\left(Y_{2}^{0}, \ldots, Y_{k}^{0}\right) \in$ $\mathcal{J}_{o}^{m}(n-1, k-1)$. Finally, the codimension of $\mathcal{A}^{m}$ in $\mathcal{J}_{o}^{m}(n, k)$ is equal to codimension of the subset $\mathcal{B}^{m}$ of all $\left(Y_{2}^{0}, \ldots, Y_{k}^{0} ; \ldots, Y_{2}^{m}, \ldots Y_{k}^{m}\right) \in \mathcal{J}_{o}^{m}(n-1, k-1) \times \mathcal{J}^{m}(n-1, m(k-$ 1)) such that:

$$
\left(\frac{\partial}{\partial x^{1}}, \sum_{\alpha=0}^{m}\left(x^{1}\right)^{\alpha} Y_{2}^{\alpha}, \ldots, \sum_{\alpha=0}^{m}\left(x^{1}\right)^{\alpha} Y_{m}^{\alpha}\right) \in \mathcal{A}^{m},
$$

in $\mathcal{J}_{o}^{m}(n-1, k-1) \times \mathcal{J}^{m}(n-1, m(k-1))$.

We study the subsystem of (16) corresponding to $i, j=2, \ldots, k$. The requirement that (15) admits a nontrivial solution $(u, \psi(\cdot))$ such that:

$$
h_{i}(\psi(t))=\mathrm{O}\left(t^{m+1}\right), \quad\left\{h_{i}, h_{j}\right\}(\psi(t))=\mathrm{O}\left(t^{m+1}\right), \quad 2 \leqslant i<j \leqslant k,
$$

defines an algebraic subset $\widehat{\mathcal{B}}^{m}$ in $\mathcal{J}_{o}^{m}(n-1, k-1) \times \mathcal{J}^{m}(n-1, m(k-1))$, where $\widehat{\mathcal{B}}^{m} \supset \mathcal{B}^{m}$. We'll show that the codimension of this larger subset tends to infinity as $m \rightarrow \infty$.

We have $x^{1}(t)=t$ in virtue of (15). We set $\eta=\left(\xi^{2}, \ldots, \xi^{n}\right), H_{i}^{\alpha}(\eta, y)=\left\langle\eta, Y_{i}^{\alpha}(y)\right\rangle$, then (15), (17) take the form:

$$
\begin{gathered}
\frac{d(\eta, y)}{d t}=\sum_{i=2}^{k} \sum_{\alpha=0}^{m} t^{\alpha} u_{i}(t) \overrightarrow{H_{i}^{\alpha}}, \\
\sum_{\alpha=0}^{m} t^{\alpha}\left\langle\eta(t), Y_{i}^{\alpha}(y(t))\right\rangle=\mathrm{O}\left(t^{m+1}\right), \\
\sum_{\alpha+\beta \leqslant m} t^{\alpha+\beta}\left\langle\eta(t),\left[Y_{i}^{\alpha}, Y_{j}^{\beta}\right](y(t))\right\rangle=\mathrm{O}\left(t^{m+1}\right), \quad 2 \leqslant i<j \leqslant k .
\end{gathered}
$$

The derivative of the function $t \mapsto\langle\eta(t), Y(y(t))\rangle$, by (18), has the form:

$$
\sum_{i=2}^{k} \sum_{\alpha=0}^{m} t^{\alpha} u_{i}(t)\left\langle\eta(t),\left[Y_{i}^{\alpha}, Y\right](y(t))\right\rangle .
$$


Successive differentiations and evaluation of the derivatives at $t=0$, show that (18), (19) are equivalent to a system of equations of the form:

$$
\begin{gathered}
\left\langle\eta(0), Y_{i}^{\alpha}(0)\right\rangle=\phi_{i}^{\alpha}\left(Y_{l}^{\beta}, u_{l}^{(\beta)}(0)\right) ; \quad \beta<\alpha, \imath=2, \ldots, k, \\
\left\langle\eta(0),\left[Y_{i}^{\alpha}(0), Y_{j}^{0}\right](0)+\left[Y_{i}^{0}(0), Y_{j}^{\alpha}\right](0)\right\rangle=\Phi_{i, j}^{\alpha}\left(Y_{l}^{\beta}, u_{l}^{(\beta)}(0)\right) ; \\
\beta<\alpha, \imath=2, \ldots, k, \quad \alpha=0,1, \ldots, m, \quad 2 \leqslant i<j \leqslant k,
\end{gathered}
$$

where $\phi_{i}^{\alpha}, \Phi_{i, j}^{\alpha}$ are certain polynomials.

The number of equations in the system (20) is $(m+1) \frac{k(k-1)}{2}$. The mappings:

$$
\left(Y_{1}^{\alpha}, \ldots, Y_{k}^{\alpha}\right) \mapsto\left(\begin{array}{c}
\left\{\left\langle\eta(0), Y_{i}^{\alpha}(0)\right\rangle\right\}_{2 \leqslant i \leqslant k} \\
\left\{\left\langle\eta(0),\left[Y_{i}^{\alpha}(0), Y_{j}^{0}\right](0)+\left[Y_{i}^{0}(0), Y_{j}^{\alpha}\right](0)\right\rangle\right\}_{2 \leqslant i<j \leqslant k}
\end{array}\right)
$$

are, obviously, submersions ( $\eta(0)$ has to be nonzero). The polynomials $\phi_{i}^{\alpha}, \Phi_{i, j}^{\alpha}$ do not depend on $Y_{l}^{\alpha}, l=1, \ldots, k$. Hence the solutions $\left(Y_{i}^{\alpha}, \eta(0), u^{(\beta)}(0)\right)$ of (20) form an algebraic subset:

$$
\mathcal{C}^{m} \subset \mathcal{J}_{o}^{m}(n-1, k-1) \times \mathcal{J}^{m}(n-1, m(k-1)) \times \mathbb{R} \mathbb{P}^{n-1} \times \mathbb{R}^{m(k-1)},
$$

of codimension $(m+1) \frac{k(k-1)}{2}$. The set $\widehat{\mathcal{B}}^{m}$ is the image of $\mathcal{C}^{m}$ under the projection:

$$
\begin{aligned}
& \mathcal{J}_{o}^{m}(n-1, k-1) \times \mathcal{J}^{m}(n-1, m(k-1)) \times \mathbb{R}^{n-1} \times \mathbb{R}^{m(k-1)} \\
& \quad \rightarrow \mathcal{J}_{o}^{m}(n-1, k-1) \times \mathcal{J}^{m}(n-1, m(k-1)) .
\end{aligned}
$$

Hence:

$$
\begin{aligned}
\operatorname{codim} \widehat{\mathcal{B}}^{m} \geqslant & (m+1) \frac{k(k-1)}{2}-(n-1)-m(k-1) \\
= & m \frac{(k-1)(k-2)}{2}-(n-1)+\frac{k(k-1)}{2} ; \\
& \operatorname{codim} \widehat{\mathcal{B}}^{m} \rightarrow \infty(m \rightarrow \infty) .
\end{aligned}
$$

Lemma 2 plus a transversality routine give the following:

COROLlaRY 4. - For any smooth manifold $N$, the set of families of distributions admitting no smooth nonzero Goh controls, contains an open everywhere dense subset of $\overline{p_{*}^{N} H_{k} T M}$.

Any smooth manifold admits a real-analytic structure and any smooth family of distributions can be approximated in the Whitney topology by a real-analytic one. What remains to be proved is that a real-analytic distribution admits a nontrivial smooth Goh control as soon as it admits a nontrivial bounded measurable Goh control. We derive this fact from the following lemma.

LEMMA 3. - Let $\dot{z}=g(z, u), z \in W, u \in U$ be a real-analytic control system and $\phi: W \times U \rightarrow \mathbb{R}^{m}$ be an analytic mapping; here $W$ is a real-analytic manifold and $U$ is a compact subanalytic set. Assume that there exists a bounded measurable control 
$u(\cdot):\left(t_{0}, t_{1}\right) \rightarrow U$ and a Lipschitzian trajectory $z(\cdot):\left(t_{0}, t_{1}\right) \rightarrow W$ such that

$$
\frac{d z}{d t}(t)=g(z(t), u(t)), \quad \phi(z(t), u(t))=0,
$$

for almost all $t \in\left(t_{0}, t_{1}\right)$. Then there also exists an analytic control $\hat{u}(\cdot):\left(\hat{t}_{0}, \hat{t}_{1}\right) \rightarrow U$ and a trajectory $\hat{z}(\cdot):\left(\hat{t}_{0}, \hat{t}_{1}\right) \rightarrow W$ such that:

$$
\frac{d \hat{z}}{d t}(t)=g(\hat{z}(t), \hat{u}(t)), \quad \phi(\hat{z}(t), \hat{u}(t))=0, \quad \forall t \in\left(\hat{t}_{0}, \hat{t}_{1}\right) .
$$

A detailed proof of this rather hard technical lemma is contained in the proof of [14, Theorem 5.1]. It follows also from anterior results by H.J. Sussmann [21,22].

The statement on real-analytic distributions we have to prove is local with respect to the state variables and we may assume that the distribution $\Delta$ under consideration is defined on $\mathbb{R}^{n}$ and admits a basis, $\Delta_{x}=\operatorname{span}\left\{X_{1}(q), \ldots, X_{k}(q)\right\}, \forall x \in \mathbb{R}^{n}$. Let $h_{i}(\xi, x)=\left\langle\xi, X_{i}(x)\right\rangle$ be the Hamiltonian associated to $X_{i}$. We set:

$$
\begin{aligned}
& W=\left(\mathbb{R}^{n *} \backslash 0\right) \times \mathbb{R}^{n}, \quad z=(\xi, x), \quad U=S^{k-1}=\left\{\left(u_{1}, \ldots, u_{k}\right) \in \mathbb{R}^{k}: \sum_{i=1}^{k} u_{i}^{2}=1\right\}, \\
& g(z, u)=\sum_{i=1}^{k} u_{i} \vec{h}_{i}(\xi, x), \quad \phi=\left(h_{1}, \ldots, h_{k} ;\left\{h_{1}, h_{2}\right\}, \ldots,\left\{h_{k-1}, h_{k}\right\}\right): W \rightarrow \mathbb{R}^{k+\frac{k(k-1)}{2}},
\end{aligned}
$$

and apply Lemma 3. Theorem 8 has been proved.

It was proved in [1, Corollary 4] that the small sub-Riemannian balls are subanalytic for any real-analytic sub-Riemannian structure without nontrivial Goh controls. Combining this fact with Theorem 8, we obtain the following result. Recall that all over the paper we keep the notation $\rho(q), q \in M$, for the sub-Riemannian distance between $q$ and the fixed point $q_{0}$. The sub-Riemannian distance is defined by a given distribution $\Delta$ on the Riemannian manifold $M$.

THEOREM 9. - Suppose that $M$ is real-analytic and $k \geqslant 3$. There exists a subset $\mathcal{A}$ of codimension $\infty$ in the space of rank $k$ real-analytic distributions on $M$ such that the relation $\Delta \notin \mathcal{A}$ implies the subanalyticity of the sub-Riemannian balls $\rho^{-1}([0, r])$ for all $r$, small enough.

\section{Nilpotent systems}

The system:

$$
\dot{x}=\sum_{i=1}^{k} u_{i}(t) Y_{i}(x), \quad x \in \mathbb{R}^{n}, x(0)=0,
$$

is called nilpotent if it coincides with its own nilpotentization expressed in adapted coordinates.

In other words, $\mathbb{R}^{n}$ is presented as a direct sum $\mathbb{R}^{n}=\mathbb{R}^{k_{1}} \oplus \cdots \oplus \mathbb{R}^{k_{l}}, k_{1}=k$, so that any vector $x \in \mathbb{R}^{n}$ takes the form $x=\left(x_{1}, \ldots, x_{l}\right), x_{i}=\left(x_{i 1}, \ldots, x_{i k_{i}}\right) \in \mathbb{R}^{k_{i}}$, 
$i=1, \ldots, l$. The vector fields $Y_{i}, i=1, \ldots, k$, are polynomial and quasi-homogeneous. More precisely, they are homogeneous of weight -1 with respect to the dilation:

$$
\delta_{t}:\left(x_{1}, x_{2}, \ldots, x_{l}\right) \mapsto\left(t x_{1}, t^{2} x_{2}, \ldots, t^{l} x_{l}\right), \quad t \in \mathbb{R} ;
$$

$\delta_{t *} Y_{i}=t Y_{i}, i=1, \ldots, k$.

We keep the notation $\hat{f}: L_{2}^{k}[0,1] \rightarrow \mathbb{R}^{n}$ for the endpoint mapping $u \mapsto x(1 ; u)$, where $x(\cdot ; u)$ is the solution of $(21), u=\left(u_{1}(\cdot), \ldots, u_{k}(\cdot)\right)$, and the notation $\hat{\rho}: \mathbb{R}^{n} \rightarrow \mathbb{R}_{+}$for the sub-Riemannian distance, $\hat{\rho}(x)=\min \{\|u\|: \hat{f}(u)=x\}$.

A special case of the system (21) with $n=l=3, k_{1}=2, k_{2}=0, k_{3}=1$, is called "the flat Martinet system". We will use the special notation $\rho^{m}: \mathbb{R}^{n} \rightarrow \mathbb{R}_{+}$for the subRiemannian distance in this case, which plays an important role below.

Proposition 7. - Assume that $k=2, k_{3} \neq 0$. Then there exists a polynomial submersion $\Phi: \mathbb{R}^{n} \rightarrow \mathbb{R}^{3}$ such that $\left(\rho^{m}\right)^{-1}([0, r])=\Phi\left(\hat{\rho}^{-1}([0, r])\right), \forall r \geqslant 0$.

Proof. - The inequality $k_{3} \neq 0$ means that at least one of the third order brackets of the fields $Y_{1}, Y_{2}$ is linearly independent on the brackets of lower order at 0 . We may assume that:

$$
\left[Y_{1},\left[Y_{1}, Y_{2}\right]\right](0) \notin \operatorname{span}\left\{Y_{1}(0), Y_{2}(0),\left[Y_{1}, Y_{2}\right](0)\right\} .
$$

There are 2 possibilities.

(1) $k_{2}=0$. Applying, if necessary a $\delta_{t}$ preserving linear change of coordinates, we may assume that $Y_{1}(0)=\partial / \partial x^{1}, Y_{2}(0)=\partial / \partial x^{2},\left[Y_{1},\left[Y_{1}, Y_{2}\right]\right](0)=\partial / \partial x^{3}$. The coordinates $x^{1}, x^{2}, x^{3}$ have the weights $1,1,3$ respectively (see Section 2 ). All other coordinates have weights not less than 3 . We have:

$$
Y_{i}(x)=\frac{\partial}{\partial x^{i}}+\sum_{j=3}^{n} b_{i}^{j}(x) \frac{\partial}{\partial x^{j}}, \quad i=1,2,
$$

where the polynomials $b_{1}^{3}(x), b_{2}^{3}(x)$ depend only on $x^{1}, x^{2}$. Then the mapping $\Phi:\left(x^{1}, \ldots, x^{n}\right) \mapsto\left(x^{1}, x^{2}, x^{3}\right)$ satisfies required properties. Indeed, $\Phi_{*} Y_{1}, \Phi_{*} Y_{2}$ are well-defined vector fields on $\mathbb{R}^{3}$ generating the flat Martinet system. Hence the image under the mapping $\Phi$ of any trajectory $t \mapsto x(t ; u)$ of the system (21) is the trajectory of the flat Martinet system associated to the same control $u$.

(2) $k_{2}=1$. We may assume that $Y_{1}(0)=\partial / \partial x^{1}, Y_{2}(0)=\partial / \partial x^{2},\left[Y_{1}, Y_{2}\right](0)=\partial / \partial x^{3}$, $\left[Y_{1},\left[Y_{1}, Y_{2}\right]\right](0)=\partial / \partial x^{4}$. The desired mapping $\Phi$ is constructed as the composition of three mappings. The first one is the projection $\Phi^{1}:\left(x^{1}, \ldots, x^{n}\right) \mapsto\left(x^{1}, \ldots, x^{4}\right)$. Then $\Phi_{*}^{1} Y_{1}, \Phi_{*}^{1} Y_{2}$ are well-defined vector fields on $\mathbb{R}^{4}$; we denote them by $Z_{i}=\Phi_{*}^{1} Y_{i}, i=$ 1,2 . The fields $Z_{1}, Z_{2}$ define a distribution $D=\operatorname{span}\left\{Z_{1}, Z_{2}\right\}$ in $\mathbb{R}^{4}$ with the growth vector $(2,3,4)$, i.e. an Engel distribution.

The Engel distribution $D$ contains a nonvanishing characteristic vector field, i.e. a vector field $Z$ such that $\left[Z, D^{2}\right]=D^{2}$. We may assume without lack of generality that $Z=Z_{2}$. This implies the relation:

$$
\left[Z_{2},\left[Z_{2}, Z_{1}\right]\right](x) \in \operatorname{span}\left\{Z_{1}(x), Z_{2}(x),\left[Z_{1}, Z_{2}\right](x)\right\} \quad \forall x \in \mathbb{R}^{4} .
$$


The vector fields $Z_{1}(x), Z_{2}(x),\left[Z_{1}, Z_{2}\right],\left[Z_{1},\left[Z_{1}, Z_{2}\right]\right]$ generate polynomial quasihomogeneous flows, thanks to their triangular "nilpotent" structure. We will use the exponential notations $e^{t Z_{1}}, e^{t Z_{2}}$, etc. for these flows. The mapping $\Phi^{2}$ is a change of coordinates $\Phi^{2}:\left(x^{1}, \ldots, x^{4}\right) \mapsto\left(y^{1}, \ldots, y^{4}\right)$, defined in the following way:

$$
\left(x^{1}, \ldots, x^{4}\right)=e^{y^{1} Z_{1}} \circ e^{y^{2} Z_{2}} \circ e^{y^{3}\left[Z_{1}, Z_{2}\right]} \circ e^{y^{4}\left[Z_{1},\left[Z_{1}, Z_{2}\right]\right]}(0) .
$$

The coordinates $\left(y^{1}, \ldots, y^{4}\right)$ are still adapted and we have:

$$
\begin{gathered}
\Phi_{*}^{2} Z_{1}=\frac{\partial}{\partial y^{1}},\left.\quad \Phi_{*}^{2} Z_{2}\right|_{y^{1}=0}=\frac{\partial}{\partial y^{2}},\left.\quad \Phi_{*}^{2}\left[Z_{1}, Z_{2}\right]\right|_{y^{1}=y^{2}=0}=\frac{\partial}{\partial y^{3}}, \\
\left.\Phi_{*}^{2}\left[Z_{1},\left[Z_{1}, Z_{2}\right]\right]\right|_{y^{1}=y^{2}=y^{3}=0}=\frac{\partial}{\partial y^{4}} .
\end{gathered}
$$

These identities and the relation (22) leave the only possibility for $\Phi_{*}^{2} Z_{2}$,

$$
\Phi_{*}^{2} Z_{2}=\frac{\partial}{\partial y^{2}}+y^{1} \frac{\partial}{\partial y^{3}}+\frac{\left(y^{1}\right)^{2}}{2} \frac{\partial}{\partial y^{4}} .
$$

In particular, the coefficients in the coordinate expression of $\Phi_{*}^{2} Z_{i}, i=1,2$, depend only on $y^{1}$.

We define $\Phi^{3}:\left(y^{1}, y^{2}, y^{3}, y^{4}\right) \mapsto\left(y^{1}, y^{2}, y^{4}\right)$ and $\Phi=\Phi^{3} \circ \Phi^{2} \circ \Phi^{1}$. The fields $\Phi_{*} Y_{1}, \Phi_{*} Y_{2}$ are well-defined and generate a flat Martinet distribution.

COROllaRY 5. - Under the conditions of Proposition 7 the sub-Riemannian balls $\hat{\rho}([0, r]), r>0$, are not subanalytic.

Proof. - Assume that $\hat{\rho}^{-1}([0, r])$ is subanalytic. Then $\Phi\left(\hat{\rho}^{-1}([0, r])\right)=\left(\rho^{m}\right)^{-1}([0, r])$ is also subanalytic because $\hat{\rho}^{-1}([0, r])$ is compact and $\Phi$ is polynomial. It is shown however in [6] that $\left(\rho^{m}\right)^{-1}([0, r])$ is not subanalytic.

Now consider nilpotent distributions of rank greater than 2, i.e. $k=k_{1}>2$. We restrict ourselves to the case of maximal possible $k_{2}, k_{3}$. It means

$$
k_{2}=\min \left\{n-k, \frac{k(k-1)}{2}\right\}, \quad k_{3}=\min \left\{n-\frac{k(k+1)}{2}, \frac{(k+1) k(k-1)}{3}\right\} .
$$

Remark. - Generic germs of distributions and their nilpotentizations have the maximal possible growth vector and, in particular, the maximal possible $k_{2}, k_{3}$.

Proposition 8. - Assume that $n \geqslant(k-1)\left(\frac{k^{2}}{3}+\frac{5 k}{6}+1\right)$ and $k_{2}, k_{3}$ are maximal possible. Then there exists a polynomial submersion $\Phi: \mathbb{R}^{n} \rightarrow \mathbb{R}^{3}$ such that $\left(\rho^{m}\right)^{-1}(r)=$ $\Phi\left(\hat{\rho}^{-1}(r)\right), \forall r \geqslant 0$.

Proof. - We'll present $\Phi$ as a composition of certain polynomial submersions. The first one is the projection:

$$
\Phi_{*}^{1}: \mathbb{R}^{n} \rightarrow \mathbb{R}^{k_{1}+k_{2}+k_{3}}, \quad \Phi^{1}(x)=\left(x_{1}, x_{2}, x_{3}\right) .
$$


Then $\Phi_{*}^{1} Y_{i}, i=1, \ldots, k$, are well-defined vector fields and the nilpotent distribution $\operatorname{span}\left\{\Phi_{*}^{1} Y_{i}: i=1, \ldots, k\right\}$ has maximal growth vector $\left(k_{1}, k_{1}+k_{2}, k_{1}+k_{2}+k_{3}\right)$ at 0 . We set $m=k_{1}+k_{2}+k_{3}, Z_{i}=\Phi_{*}^{1} Y_{i}, D_{x}=D_{x}^{1}=\operatorname{span}\left\{Z_{i}(x): 1 \leqslant i \leqslant k\right\}$,

$$
\begin{aligned}
& D_{x}^{2}=\operatorname{span}\left\{\left[Z_{i}, Z_{j}\right](x): 1 \leqslant i, j \leqslant k\right\}, \\
& D_{x}^{3}=\operatorname{span}\left\{\left[Z_{l},\left[Z_{i}, Z_{j}\right]\right](x): 1 \leqslant i, j, l \leqslant k\right\} .
\end{aligned}
$$

The maximality of $k_{2}, k_{3}$ and homogeneity of $Z_{i}$ with respect to the dilation imply that $\operatorname{dim} D_{x}^{i}=k_{i}, i=1,2,3, \forall x \in \mathbb{R}^{n}$.

Take bracket monomials:

$$
Z_{k_{1}+\alpha}=\left[Z_{i_{\alpha 1}}, Z_{i_{\alpha 2}}\right], \quad Z_{k_{1}+k_{2}+\beta}=\left[Z_{i_{\beta 1}},\left[Z_{i_{\beta 2}}, Z_{i_{\beta 3}}\right]\right],
$$

$\alpha=1, \ldots, k_{2}, \beta=1, \ldots, k_{3}, 1 \leqslant i_{\alpha j}, i_{\beta j} \leqslant k_{1}$, in such a way that $Z_{1}(0), \ldots, Z_{m}(0)$ form a basis of $\mathbb{R}^{m}$. Then $Z_{1}(x), \ldots, Z_{m}(x)$ form a basis of $\mathbb{R}^{m}$ for $\forall x \in \mathbb{R}^{m}$. In particular, any Lie monomial of the fields $Z_{1}, \ldots, Z_{k}$ is a linear combination of the fields $Z_{1}, \ldots, Z_{m}$ with smooth coefficients. The nilpotency of the system $Z_{1}, \ldots, Z_{k}$ implies that these coefficients have weight 0 and are actually constants. Moreover, all Lie monomials of order greater than 3 are zero. We obtain that the fields $Z_{1}, \ldots, Z_{k}$ generate an $m$-dimensional nilpotent Lie algebra with the basis $Z_{1}, \ldots, Z_{m}$; the sub-Riemannian structure with the orthonormal frame $Z_{1}, \ldots, Z_{k}$ is isometric to the left-invariant subRiemannian structure on the corresponding $m$-dimensional simply connected nilpotent Lie group $G_{m}$. We will identify $G_{m}$ with $\mathbb{R}^{m}$ and assume that the fields $Z_{i}$ are leftinvariant.

LeMmA 4. - Let $I\left(Z_{3}, \ldots, Z_{k}\right)$ be the ideal in the Lie algebra $\operatorname{Lie}\left\{Z_{1}, \ldots, Z_{k}\right\}$ generated by $Z_{3}, \ldots, Z_{k}$. If $\operatorname{dim}\left(\operatorname{Lie}\left\{Z_{1}, \ldots, Z_{k}\right\}\right) \geqslant(k-1)\left(\frac{k^{2}}{3}+\frac{5 k}{6}+1\right)$, then $\operatorname{dim}\left(\operatorname{Lie}\left\{Z_{1}, \ldots, Z_{k}\right\} / I\left(Z_{3}, \ldots, Z_{k}\right)\right) \geqslant 4$.

Proof. - The following monomials represent the specialization of a Ph. Hall basis of the free Lie algebra with $k$ generators up to the order 3:

$$
Z_{i},\left[Z_{i}, Z_{j}\right],\left[Z_{l},\left[Z_{i}, Z_{j}\right]\right], \quad i, j, l \in\{1, \ldots, k\}, i<j, i \leqslant l .
$$

This $\mathrm{Ph}$. Hall basis consists of

$$
v_{3}(k)=k+\frac{k(k-1)}{2}+\frac{(k+1) k(k-1)}{3}=(k-1)\left(\frac{k^{2}}{3}+\frac{5 k}{6}+1\right)+1
$$

elements. Hence $m$ equals either $\nu_{3}(k)$ or $v_{3}(k)-1$. In both cases, removing the fields $\left[Z_{1},\left[Z_{1}, Z_{2}\right]\right],\left[Z_{2},\left[Z_{2}, Z_{1}\right]\right]$ from the list (23) we obtain that the linear hull of the remaining fields is a proper subspace of $\operatorname{Lie}\left\{Z_{1}, \ldots, Z_{k}\right\}$.

Let $\phi: \operatorname{Lie}\left\{Z_{1}, \ldots, Z_{k}\right\} \rightarrow \operatorname{Lie}\left\{Z_{1}, \ldots, Z_{k}\right\} / I\left(Z_{3}, \ldots, Z_{k}\right)$ be the canonical homomorphism. We obtain that at least one of the fields $\phi\left(\left[Z_{1},\left[Z_{1}, Z_{2}\right]\right]\right), \phi\left(\left[Z_{2},\left[Z_{2}, Z_{1}\right]\right]\right)$ is nonzero.

Let $G(I)$ be the normal subgroup of $G_{m}$ generated by $I\left(Z_{3}, \ldots, Z_{k}\right)$. Then $\phi=$ $\Phi_{*}^{2}$, where $\Phi^{2}: G_{m} \rightarrow G_{m} / G(I)$ is the canonical epimorphism. We have $\Phi_{*}^{2} Z_{3}=$ 
$\cdots=\Phi_{*}^{2} Z_{n}=0$, while $\operatorname{span}\left\{\Phi_{*}^{2} Z_{1}, \Phi_{*}^{2} Z_{2}\right\}$ is a nilpotent distribution with the growth vector $2,3,5$ or $2,3,4$. We are thus in the situation of Proposition 7. This proposition provides us with the submersion $\Phi^{3}: G_{m} / G(I) \rightarrow \mathbb{R}^{3}$ which "projects" the subRiemannian structure with orthonormal frame $\Phi_{*}^{2} Z_{1}, \Phi_{*}^{2} Z_{2}$ onto the flat Martinet structure. Finally, we set $\Phi=\Phi^{3} \circ \Phi^{2} \circ \Phi^{1}$.

COROLlaRY 6. - Under the conditions of Proposition 8, the sub-Riemannian balls $\hat{\rho}([0, r]), r>0$, are not sub-analytic.

The proof is a strict repetition of the proof of Corollary 5 .

Let now $\Delta$ be an arbitrary (not necessarily nilpotent) germ of a bracket generating distribution at $q_{0} \in M$, and let $\rho$ be the germ of the associated sub-Riemannian distance function. Combining Corollaries 5, 6, and Theorem 4 we obtain the following:

THEOREM 10. - Assume that either $k=2$ and $\Delta_{q_{0}}^{3} \neq \Delta_{q_{0}}^{2}$ or $\operatorname{dim} M \geqslant(k-1)\left(\frac{k^{2}}{3}+\right.$ $\left.\frac{5 k}{6}+1\right)$ and the segment $\left(k, \operatorname{dim} \Delta_{q_{0}}^{2}, \operatorname{dim} \Delta_{q_{0}}^{3}\right)$ of the growth vector is maximal. Then $\rho$ is not subanalytic. In particular, generic germs are such that $\rho$ is not subanalytic.

Finally, combining Theorem 10 with Theorem 9 we come to the following surprising result.

COROLLARY 7. - Let $\rho$ be a germ of sub-Riemannian distance function associated with a generic germ of real-analytic distribution of rank $k \geqslant 3$, on a $n$-dimensional manifold, $n \geqslant(k-1)\left(\frac{k^{2}}{3}+\frac{5 k}{6}+1\right)$. Then the balls $\rho^{-1}([0, r])$ are subanalytic for all small enough $r$, but the function $\rho$ is not subanalytic!

\section{Acknowledgement}

We are grateful to Sébastien Jacquet for fruitful discussions.

\section{REFERENCES}

[1] Agrachev A.A., Compactness for sub-Riemannian length-minimizers and subanalyticity, Rend. Semin. Mat. Torino 56 (1998).

[2] Agrachev A.A., Gamkrelidze R.V., Sarychev A.V., Local invariants of smooth control systems, Acta Appl. Math. 14 (1989) 191-237.

[3] Agrachev A.A., Sarychev A.V., Filtrations of a Lie algebra of vector fields and nilpotent approximation of control systems, Dokl. Akad. Nauk SSSR 295 (1987) 777-781; English transl. in Soviet Math. Dokl. 36 (1988) 104-108.

[4] Agrachev A.A., Sarychev A.V., Abnormal sub-Riemannian geodesics: Morse index and rigidity, Annales de l'Institut Henri Poincaré, Analyse non linéaire 13 (1996) 635-690.

[5] Agrachev A.A., Sarychev A.V., Sub-Riemannian metrics: minimality of abnormal geodesics versus subanalyticity, J. ESAIM: Control, Optimisation and Calculus of Variations 4 (1999) 377-403.

[6] Agrachev A.A., Bonnard B., Chyba M., Kupka I., Sub-Riemannian sphere in Martinet flat case, J. ESAIM: Control, Optimisation and Calculus of Variations 2 (1997) 377-448.

[7] Bellaïche A., The tangent space in sub-Riemannian geometry, in: Sub-Riemannian Geometry, Birkhäuser, 1996, pp. 1-78. 
[8] Bianchini R.M., Stefani G., Graded approximations and controllability along a trajectory, SIAM J. Control Optim. 28 (1990) 903-924.

[9] Bonnard B., Chyba M., Méthodes géométriques et analytique pour étudier l'application exponentiele, la sphère et le front d'onde en géometrie SR dans le cas Martinet, J. ESAIM: Control, Optimisation and Calculus of Variations, submitted.

[10] Bonnard B., Launay G., Trélat E., The transcendence we need to compute the sphere and the wave front in Martinet SR-geometry, in: Proc. Int. Confer. Dedicated to Pontryagin, Moscow, September 1998, to appear.

[11] Chow W.-L., Über Systeme von linearen partiellen Differentialgleichungen ester Ordnung, Math. Ann. 117 (1939) 98-105.

[12] Filippov A.F., On certain questions in the theory of optimal control, Vestnik Moskov. Univ., Ser. Matem., Mekhan., Astron. 2 (1959) 25-32.

[13] Gabrielov A., Projections of semi analytic sets, Funct. Anal. Appl. 2 (1968) 282-291.

[14] Gauthier J.-P., Kupka I., Observability for systems with more outputs than inputs and asymptotic observers, Mathem. Zeitschrift 223 (1996) 47-78.

[15] Ge Zhong, Horizontal path space and Carnot-Caratheodory metric, Pacific J. Math. 161 (1993) 255-286.

[16] Hironaka H., Subanalytic sets, in: Numbers Theory, Algebraic Geometry, and Commutative Algebra (in honor of V. Akizuki), Tokyo, 1973, pp. 453-493.

[17] Jacquet S., Subanalyticity of the sub-Riemannian distance, J. Dynamical and Control Systems 5 (1999).

[18] Rashevsky P.K., About connecting two points of a completely nonholonomic space by admissible curve, Uch. Zapiski Ped. Inst. Libknechta 2 (1938) 83-94.

[19] Stefani G., On local controllability of a scalar-input system, in: Byrnes, Lindquist (Eds.), Theory and Appl. of Nonlinear Control Syst., North Holland, Amsterdam, 1986, pp. 167179.

[20] Sussmann H.J., Optimal control and piecewise analyticity of the distance function, in: Ioffe A., Reich S. (Eds.), Pitman Research Notes in Mathematics, Longman Publishers, 1992, pp. 298-310.

[21] Sussmann H.J., Trajectory regularity and real analyticity, in: Proc. 25th CDC Conference, Athens, Greece, 1986, pp. 592-595.

[22] Sussmann H.J., A weak regularity theorem for real-analytic optimal control problems, Revista Mathematica Iberoamericana 2 (3) (1986) 307-317.

[23] Tamm M., Subanalytic sets in the calculus of variations, Acta Mathematica 46 (1981) 167199. 